\title{
Microcanonical and finite-temperature $a b$ initio molecular dynamics simulations on quantum computers
}

\author{
Igor O. Sokolov $\odot,{ }^{1,2}$ Panagiotis Kl. Barkoutsos, ${ }^{1}$ Lukas Moeller, ${ }^{1}$ Philippe Suchsland, ${ }^{1,3}$ \\ Guglielmo Mazzola, ${ }^{1}$ and Ivano Tavernelli ${ }^{1, *}$ \\ ${ }^{1}$ IBM Quantum, IBM Research-Zurich, Switzerland \\ ${ }^{2}$ Department of Chemistry, University of Zurich, Winterthurerstrasse 190, 8057 Zurich, Switzerland \\ ${ }^{3}$ Department of Physics, University of Zurich, Winterthurerstrasse 190, 8057 Zurich, Switzerland
}

(Received 24 August 2020; revised 19 October 2020; accepted 17 November 2020; published 10 February 2021)

\begin{abstract}
$A b$ initio molecular dynamics (AIMD) is a powerful tool to predict properties of molecular and condensed matter systems. However, the quality of this procedure relies on the availability of rigorous electronic structure calculations. The development of quantum processors has shown great potential for the efficient evaluation of accurate ground and excited state energies of molecular systems, opening up new avenues for molecular dynamics simulations. In this work, we address the use of variational quantum algorithms for the calculation of accurate atomic forces to be used in AIMD. In particular, we provide solutions for the alleviation of the statistical noise associated with the measurements of the expectation values of energies and forces, as well as schemes for the mitigation of the hardware noise sources (in particular, gate infidelities, qubit decoherence, and readout errors). Despite the relative large error in the calculation of the potential energy, our results show that the proposed algorithms can provide accurate MD trajectories in the microcanonical (constant energy) ensemble. Furthermore, exploiting the intrinsic noise associated to the quantum measurement process, we also propose a Langevin dynamics algorithm for the simulation of canonical, i.e., constant temperature, dynamics. Both algorithms (microcanonical and canonical) are applied to the simulation of simple molecular systems such as $\mathrm{H}_{2}$ and $\mathrm{H}_{3}^{+}$. Finally, we also provide results for the dynamics of $\mathrm{H}_{2}$ obtained with IBM quantum computer ibmq_athens.
\end{abstract}

DOI: 10.1103/PhysRevResearch.3.013125

\section{INTRODUCTION}

Quantum computing is emerging as a new computational paradigm for the solution, among others, of quantum mechanical many-body problems. In particular, in recent years we have witnessed a rapid development of quantum algorithms for electronic structure calculations of both ground and excited states properties [1-3] as well as vibrational structure calculations [4-6]. The variational quantum eigensolver (VQE) algorithm [7] allows for the efficient calculation of the electronic structure of simple molecules both in simulations as well as in hardware experiments [1-3,8-19]. Typically, in the VQE algorithm, the molecular wave function is encoded in the quantum register using a classically inspired wave function ansatz, such as unitary coupled cluster $[10,20]$, or by means of a "heuristic" expansion dictated by the available gates and connectivity in the hardware [11].

In all cases, it is possible to show that a polynomial number of variational parameters (qubit rotations) are sufficient to achieve accurate results within the so-called chemical accuracy (i.e., with an error less than $1 \mathrm{kcal} / \mathrm{mol}$ or $1.6 \times 10^{-3}$

\footnotetext{
*ita@zurich.ibm.com

Published by the American Physical Society under the terms of the Creative Commons Attribution 4.0 International license. Further distribution of this work must maintain attribution to the author(s) and the published article's title, journal citation, and DOI.
}

hartree) $[21,22]$. Of particular relevance is the theoretical scaling of these new algorithms, which corresponds to $O\left(N_{b}^{4}\right)$ in the number of basis functions $N_{b}$. On the other hand, the accuracy of the results depends on the nature of the wave function ansatz mapped in the qubit space, which needs to offer the best possible representability of the ground state wave function $[1,23,24]$. It is worth mentioning that despite the potential advantage of the quantum algorithms, the simulation of the quantum circuits is memory limited on classical computers due to the exponential scaling of the Hilbert space in the number of qubits, while state-of-the-art quantum hardware is still too noisy to achieve chemical accuracy on shallow circuits.

In addition to the calculation of energies, quantum algorithms can provide an efficient solution to the calculation of the $a b$ initio forces on the classical, pointlike, atomic particles. These are of particular importance for the calculation of optimized molecular structures (through annealing) as well as to perform molecular dynamics (MD) in the different thermodynamic ensembles. In fact, the quantum circuit optimized for the calculation of the ground state energy can also be used for the evaluation of the expectation values of the gradients of the Hamiltonian with respect to the nuclear coordinates, giving access to the nuclear forces. Several approaches have been already described in the literature. The most direct approach relies on finite difference (FD) approximations [25-27], which can lead to fairly accurate forces at the cost of $6 \mathrm{~N}$ additional wave function optimizations with 
$N$ being the number of nuclei. To reduce this overhead, one can also make use of a modified FD approach based on the Hellmann-Feynman theorem, which only requires a single wave function evaluation as shown in Refs. [26-28]; this will also be the method of choice in this work. Alternatively, one can also use analytic gradients in the framework of VQE [27] or the Lagrangian formalism, which allows the determination of the response functions with respect to the nuclear displacement without the explicit calculation of the derivatives [29].

In this work, we investigate quantum algorithms for the calculation of atomic forces to perform molecular dynamics in the microcanonical NVE ensemble (i.e., constant number of particles, volume, and energy) as well as in the constant temperature canonical NVT ensemble (i.e., constant number of particles, volume, and temperature). In the first case, we use a well-studied classical integration scheme of the Newton's equations of motion, namely the Verlet algorithm [30] to compute constant energy trajectories using VQE energies and forces. In the second case, we make use of the intrinsic statistical noise in evaluating quantum observables in a quantum computer to perform generalized Langevin dynamics [31,32] at constant (nonzero) temperature. Both approaches are applied to the simulation of the dynamics of simple molecular systems such as $\mathrm{H}_{2}$ and $\mathrm{H}_{3}^{+}$.

The dynamics is performed using both the matrix representation (MR) of the parametrized VQE circuit as well as the classical emulation of the VQE algorithm, as it would be executed on a quantum computer. In this case, a realistic representation of the hardware noise, including fidelity of the qubit operations, qubit decoherence, and readout errors, is applied to mimic as close as possible the hardware conditions. Finally, for the $\mathrm{H}_{2}$ molecule, we also perform a study of the dynamics in the NVE ensemble on a noisy quantum computer with the aim to demonstrate the feasibility, but also the current limitations, of our approach.

The main goal of this work remains the study of quantum algorithms for the calculation of forces in the presence of realistic noise models. In particular, we focus on the requirements in terms of qubit fidelity, decoherence, and measurement error rates necessary to obtain accurate trajectories for near-term quantum calculations. We investigate the impact of the noise on the determination of the system energy and on the direct evaluation of the forces, showing evidences for a stable MD scheme based on a modified Hellmann-Feynman approach that, while approximated, can lead to stable dynamics. Due to the dominant role of the noise, in this work we do not explore other more sophisticated solutions [15,33-36], which improve the accuracy of the formal derivation of the forces at the cost of increasing the computational requirements. However, to mitigate the impact of the noise, we also apply an error mitigation scheme based on the Lanczos algorithm [37] and on the power iteration method [38], which improves substantially the quality of the energies and forces without modifying the overall scaling of the MD algorithm.

This paper is organized as follows. In Sec. II we define the molecular Hamiltonian and discuss reusability of measurements for the calculation of energies and forces, using the centered finite differences and the Hellmann-Feynman approaches. To mitigate the effect of noise, the Lanczos method is introduced. In Sec. III, we discuss the MD schemes for microcanonical dynamics using the Verlet algorithm and, for canonical ensembles, the generalized Langevin dynamics that exploits the statistical noise associated with the quantum measurements process. The results are presented in Sec. IV where we first show the outcomes of the geometry optimizations and then of the microcanonical MD simulations of the $\mathrm{H}_{2}$ and $\mathrm{H}_{3}^{+}$molecules. In the case of $\mathrm{H}_{2}$, we also present a microcanonical MD trajectory obtained on a quantum computer. Finally, the Langevin dynamics simulations demonstrate how the canonical distribution (Boltzmann) can also be achieved. Conclusions are presented in Sec. V.

\section{CALCULATION OF FORCES}

\section{A. Electronic ground state calculation}

The basis of our approach is the Born-Oppenheimer approximation [39], which allows to separate the dynamics of the electronic and nuclear subsystems. More specifically, we constrain the ionic dynamics to adiabatically follow the potential energy surface (PES) defined by solution of the instantaneous electronic ground state at fixed ionic positions.

As standard practice in state-of-the-art electronic structure calculations in quantum computers [1-3], we adopt the second quantized formulation of the molecular Hamiltonian

$$
\begin{aligned}
\hat{H}(\boldsymbol{R})= & \sum_{r s} h_{r s}(\boldsymbol{R}) \hat{a}_{r}^{\dagger} \hat{a}_{s} \\
& +\frac{1}{2} \sum_{p q r s} g_{p q r s}(\boldsymbol{R}) \hat{a}_{p}^{\dagger} \hat{a}_{q}^{\dagger} \hat{a}_{r} \hat{a}_{s}+E_{N N}(\boldsymbol{R}),
\end{aligned}
$$

with $h_{r s}(\boldsymbol{R})$ denoting the one-electron integrals and $g_{p q r s}(\boldsymbol{R})$ denoting the two-electron integrals in physics notation (see Appendix A), which are commonly obtained with a HartreeFock (HF) calculation. Notice that the collective vector of nuclear coordinates $\boldsymbol{R}=\left(\boldsymbol{R}_{1}, \boldsymbol{R}_{2}, \ldots, \boldsymbol{R}_{N}\right)$ of $N$ nuclei in $\mathbb{R}^{3 N}$ simply parametrizes the electronic Hamiltonian. The position vector of a single nuclei $I \in\{1, \ldots, N\}$ is denoted by $\boldsymbol{R}_{I}=$ $\left(R_{I x}, R_{I y}, R_{I z}\right)$. The operators $\hat{a}_{r}^{\dagger}\left(\hat{a}_{r}\right)$ represent the fermionic creation (annihilation) operators for electrons in HF spin orbitals (MOs). The indices $p, q, r, s$ are used to label general (occupied or virtual) MOs. The term $E_{N N}(\boldsymbol{R})$ describes the nuclear repulsion energy. A complete description of the terms in Eq. (1) is given in Appendix A.

The enabling step of the approach is to find first the ground state of Eq. (1). While in general this task is not achievable exactly, a close approximation of the ground state can be obtained variationally by using the VQE algorithm [21]. This method features quantum circuits with gates that are defined collectively by optimizable parameters $\boldsymbol{\theta}$. This generates a parametrized quantum state $|\Psi(\theta)\rangle$, often called trial state. These parameters are optimized to minimize the energy $E(\boldsymbol{\theta})=\langle\Psi(\boldsymbol{\theta})|\hat{H}(\boldsymbol{R})| \Psi(\boldsymbol{\theta})\rangle$ for a given Hamiltonian. This optimization is performed classically. Since this approach is well-established, we direct the reader to Refs. [7,40] for further details.

Of particular relevance for MD is the accurate estimation of expectation values of the form

$$
\langle\hat{\mathcal{O}}\rangle=\langle\Psi(\boldsymbol{\theta})|\hat{\mathcal{O}}| \Psi(\boldsymbol{\theta})\rangle
$$


for a generic operator $\hat{\mathcal{O}}$. Formally, any Hermitian operator $\hat{\mathcal{O}}$ defined on an $n$-qubit Hilbert space can be represented as

$$
\hat{\mathcal{O}}=\sum_{\lambda=1}^{\Lambda} c_{\lambda} \hat{P}_{\lambda}, \quad c_{\lambda} \in \mathbb{R} .
$$

Each of the $\Lambda n$-qubit Pauli strings is an element of the set $\mathcal{P}_{n}=\left\{\hat{p}_{1} \otimes \hat{p}_{2} \otimes \cdots \otimes \hat{p}_{n} \mid \hat{p}_{i} \in\{\hat{I}, \hat{X}, \hat{Y}, \hat{Z}\}\right\}$, and is tensor products of $n$ single qubit Pauli operators (see Appendix B).

The expectation value in Eq. (2) is calculated as the sum of the expectation values $\left\langle\hat{P}_{\lambda}\right\rangle$ of the single Pauli operator, multiplied by the respective scalar number $c_{\lambda}$. Finally, the expectation value $\left\langle\hat{P}_{\lambda}\right\rangle$ can be obtained by sampling from the prepared state $|\Psi\rangle$ using $\mathcal{N}$ measurements, hence $\mathcal{N}$ repetition of the same circuit (see [8] for details). The statistical error associated with the evaluation of $\left\langle\hat{P}_{\lambda}\right\rangle$ decreases as $1 / \sqrt{\mathcal{N}}$. The total number of measurements required to compute $\langle\hat{\mathcal{O}}\rangle$ is therefore $\Lambda \mathcal{N}$, assuming for simplicity to allocate the same number of resources for each Pauli string. While extensive algorithmic efforts have been recently put forward to mitigate this issue [8,41-45], the impact of the statistical noise in evaluating quantum observables remains a peculiar aspect of quantum computation.

In the case of the energy, the target qubit operator $\hat{\mathcal{O}}$ is the Hamiltonian of Eq. (1) to which we apply the Jordan-Wigner transformation [46] (for instance) that maps the fermionic operators $\hat{a}_{r}^{\dagger}$ and $\hat{a}_{r}$ to qubit operators. The size of the qubit register $n$ is defined by the maximum number of molecular spin orbitals considered in Eq. (1).

We now consider the case in which we need to measure not just one but several, operators $\hat{\mathcal{O}}_{i}$ (with $i=1, \ldots, 3 N$ ) which share the same support in the set $\mathcal{P}_{n}$ and only differ in the values $\left\{c_{\lambda}^{(i)}\right\}$. These $3 N$ operators can therefore be computed from the same data set of $\Lambda \mathcal{N}$ circuit measurements. The expectation values of these operators become correlated and the corresponding covariance matrix is defined as

$$
\operatorname{Cov}_{i j}=\left\langle\left(\hat{\mathcal{O}}_{i}-\left\langle\hat{\mathcal{O}}_{i}\right\rangle\right)\left(\hat{\mathcal{O}}_{j}-\left\langle\hat{\mathcal{O}}_{j}\right\rangle\right)\right\rangle .
$$

This concept will become useful in the following discussion (see Sec. III B) where the covariance matrix is built for the force operators [Eq. (18)] and used to drive the molecular dynamics in the canonical ensemble.

\section{B. Force estimator}

The forces on the nuclei $\boldsymbol{F}=\left(\boldsymbol{F}_{1}, \boldsymbol{F}_{2}, \ldots, \boldsymbol{F}_{N}\right)$ with $\boldsymbol{F}_{I}=$ $\left(F_{I x}, F_{I y}, F_{I z}\right)$ are the derivatives of the energy with respect to the nuclear coordinates $F_{I \alpha}(\boldsymbol{R})=\left.\frac{\mathrm{d} E}{\mathrm{~d} R_{I \alpha}}\right|_{\boldsymbol{R}}$, where $\alpha \in\{x, y, z\}$. The total derivative is given explicitly by

$$
\begin{aligned}
F_{I \alpha}(\boldsymbol{R})= & \left\langle\Psi(\boldsymbol{\theta})\left|\partial_{I \alpha} \hat{H}(\boldsymbol{R})\right| \Psi(\boldsymbol{\theta})\right\rangle \\
& +\left\langle\partial_{I \alpha} \Psi(\boldsymbol{\theta})|\hat{H}(\boldsymbol{R})| \Psi(\boldsymbol{\theta})\right\rangle \\
& +\left\langle\Psi(\boldsymbol{\theta})|\hat{H}(\boldsymbol{R})| \partial_{I \alpha} \Psi(\boldsymbol{\theta})\right\rangle,
\end{aligned}
$$

with $\partial_{I \alpha}=\frac{\partial}{\partial R_{I \alpha}}$, where the second and last terms denote the wave function contributions, the so-called Pulay forces [47] (see Ref. [48] for possible implementations in a quantum circuit). The first term corresponds to the Hellmann-Feynman force [49-52].
For an approximation of this derivative (see Refs. [26,27]), the centered FD method can be applied as

$$
F_{I \alpha}^{\mathrm{FD}}(\boldsymbol{R})=\frac{\left\langle\Psi_{+}\left|\hat{H}_{+}\right| \Psi_{+}\right\rangle-\left\langle\Psi_{-}\left|\hat{H}_{-}\right| \Psi_{-}\right\rangle}{2 \Delta R},
$$

where $\hat{H}_{ \pm}=\hat{H}\left(\boldsymbol{R} \pm \Delta \boldsymbol{R}_{I \alpha}\right)$ with corresponding ground states $\left|\Psi_{ \pm}\right\rangle$evaluated, respectively, at $\boldsymbol{R} \pm \Delta \boldsymbol{R e}_{I \alpha}\left(\boldsymbol{e}_{I \alpha}\right.$ is a directional unit vector and $\Delta R$ is the step size). In order to compute all molecular forces $\boldsymbol{F}$, we therefore need to perform $6 \mathrm{~N}$ electronic structure calculations (e.g., using VQE) to obtain the states $\left|\Psi_{ \pm}\right\rangle$, making this approach computationally costly. In addition, the centered FD method typically introduces sizable numerical errors, i.e., round-off and discretization errors, with the latter scaling with the step size as $O\left(\Delta R^{2}\right)$. In the noisy setting, this approach suffers from large errors due to independent statistical errors in the computations of the left and right expectation values in Eq. (6). This is sufficient to limit its practical implementation [27] which, at variance with quantum Monte Carlo methods, cannot be improved by using correlated sampling techniques [53].

Therefore, in this work we opt for the Hellmann-Feynman approach (see also O'Brien et al. [28]), which consists in considering only the first term in Eq. (5) and applying numerical differentiation to the term $\partial_{I \alpha} \hat{H}(\boldsymbol{R})$ as

$$
F_{I \alpha}^{\mathrm{H}-\mathrm{F}}(\boldsymbol{R})=\left\langle\Psi_{0}\left|\frac{\hat{H}_{+}-\hat{H}_{-}}{2 \Delta R}\right| \Psi_{0}\right\rangle,
$$

where $\left|\Psi_{0}\right\rangle$ is the optimized ground state wave function at geometry $\boldsymbol{R}$. In contrast to the standard FD approach described in Eq. (6), only a single ground state wave function $\left|\Psi_{0}\right\rangle$ is needed to calculate all $\boldsymbol{F}^{\mathrm{H}-\mathrm{F}}$ components, of $6 N\left|\Psi_{ \pm}\right\rangle$wave functions required for $\boldsymbol{F}^{\mathrm{FD}}$. Therefore, within the HellmannFeynman approach, the calculation of forces requires the same measurements as for the ground state calculation. The only difference lies in the values of the coefficients (i.e., the classical integrals) associated with different Pauli strings, which in the case of forces are evaluated (classically) at the displaced geometries [28]. The error due to this approximation is given by the Pulay force term $\Delta F_{\text {corr }, I \alpha}=\left\langle\partial_{I \alpha} \Psi(\boldsymbol{\theta})|\hat{H}(\boldsymbol{R})| \Psi(\boldsymbol{\theta})\right\rangle+$ $\left\langle\Psi(\boldsymbol{\theta})|\hat{H}(\boldsymbol{R})| \partial_{I \alpha} \Psi(\boldsymbol{\theta})\right\rangle$ assuming that $\Delta R$ is chosen sufficiently small to minimize the contribution of the discretization error. It is worth mentioning that a perturbation-dependent basis can also be constructed such that forces can be computed solely from the Hellmann-Feynman term at the cost of incorporating reorthonormalization operations of the orbitals in the Hamiltonian as described in Ref. [54]. Note that the use of numerical differentiation in the evaluation of the H-F forces [Eq. (7)] can be overcome altogether by applying the exact analytic derivatives of the one-/two-body integrals (see the Supplemental Material of Ref. [28]).

\section{Statistical and hardware noise sources}

In this section, we briefly summarize the different error types occurring in the implementation of the force algorithm on a quantum computer. The first error source, which we name systematic, stems from the approximate solution of the ground state electronic wave function for the Hamiltonian in Eq. (1). This error can be reduced systematically by employing more accurate trial wave functions, which can better represent the 
true ground state at fixed $\boldsymbol{R}$, as well as by improving the (classical) optimization algorithm in the VQE approach.

The second error type originates from the statistical evaluation of the force operators of Eq. (6) with a finite number of measurements and we name it statistical noise. This issue has been already discussed in Sec. II A and cannot be alleviated even when using the exact ground state wave function. However, in Sec. III B we will show how we can exploit statistical noise to perform finite-temperature MD simulations.

Finally, the last source of errors is due to hardware noise. This is modeled by the gate, readout, and thermalization errors represented by Kraus operators that are applied to the density matrix [55]. Gate and thermalization errors are introduced as depolarization and general amplitude damping channels. The gate and thermalization errors can be loosely categorized as systematic as they affect the possibility to reach the ground state. The readout noise instead is hard to model and cannot easily be absorbed in the statistical noise contribution. For a more detailed account on the implementation of these noise types in Qiskit [56] see Appendix C.

\section{Lanczos noise mitigation scheme}

A method to mitigate the noise of near-term quantum computers consists in applying the Lanczos mitigation scheme for the evaluation of the expectation value of operators in the VQE algorithm. The evaluation of the expectation value of a given operator $\hat{\mathcal{O}}$ with the VQE wave function $\left|\Psi_{0}\right\rangle$ is affected by hardware noise and measurement errors. Strictly speaking, this implies that, under noisy conditions, also higher "exact" eigenstates of the Hamiltonian $\hat{H}(\boldsymbol{R})$ will contribute to the evaluation of the expectation value $\left\langle\Psi_{0}|\hat{\mathcal{O}}| \Psi_{0}\right\rangle$. In order to alleviate this effect, we propose to use the Lanczos approach $[57,58]$, which has the effect of partially projecting out the contributions of the excited states from the measurement of the expectation values, reducing therefore the effect of noise. When referring to the Lanczos mitigation scheme in the VQE$\mathrm{L}$ algorithm, we will imply the substitution

$$
\begin{aligned}
\left\langle\Psi_{0}|\hat{\mathcal{O}}| \Psi_{0}\right\rangle & \rightarrow \frac{\left\langle\Psi_{0}|(\hat{H}(\boldsymbol{R})-d) \hat{\mathcal{O}}(\hat{H}(\boldsymbol{R})-d)| \Psi_{0}\right\rangle}{\left\langle\Psi_{0}\left|(\hat{H}(\boldsymbol{R})-d)^{2}\right| \Psi_{0}\right\rangle} \\
& =L_{d, \boldsymbol{R}}(\hat{\mathcal{O}}),
\end{aligned}
$$

where $d \in \mathbb{R}$ is a tunable parameter that needs to be optimized a priori (see Appendix D).

Note that the increase in accuracy is obtained at the cost of additional measurements of the terms in Eq. (8), which include expectation values of $\hat{H}(\boldsymbol{R}) \hat{\mathcal{O}} \hat{H}(\boldsymbol{R})$.

The method can be rationalized by interpreting $L_{d, \boldsymbol{R}}(\hat{\mathcal{O}})$ as the measurement of $\hat{\mathcal{O}}$ with respect to the modified state

$$
\left|\Psi_{0}\right\rangle \rightarrow \frac{(\hat{H}(\boldsymbol{R})-d)\left|\Psi_{0}\right\rangle}{N_{\Psi_{0}}}=\sum_{i} \frac{\left(E_{i}(\boldsymbol{R})-d\right)\left|E_{i}(\boldsymbol{R})\right\rangle \alpha_{i}}{N_{\Psi_{0}}}
$$

where we used the spectral representation of $\hat{H}(\boldsymbol{R})$ in its eigenstates $\left\{\left|E_{i}(\boldsymbol{R})\right\rangle\right\}$ with $\left|E_{0}(\boldsymbol{R})\right\rangle$ the true ground state approximated by $\left|\Psi_{0}\right\rangle, N_{\Psi_{0}}^{2}=\left\langle\Psi_{0}\left|(\hat{H}(\boldsymbol{R})-d)^{2}\right| \Psi_{0}\right\rangle$ and the projection $\alpha_{i}=\left\langle E_{i}(\boldsymbol{R}) \mid \Psi_{0}\right\rangle$. The coefficient associated with
$\left|E_{i}(\boldsymbol{R})\right\rangle$ is selected to fulfill the condition

$$
\frac{\left|E_{0}(\boldsymbol{R})-d\right|}{N_{\Psi_{0}}}>1
$$

So that to increase the contribution of the ground state. In fact, by enforcing $\left|L_{d, \boldsymbol{R}}(\hat{H}(\boldsymbol{R}))-d\right| / N_{\Psi_{0}}>1$ and $d>L_{d, \boldsymbol{R}}(\hat{H}(\boldsymbol{R}))$ one gets that $\left|E_{0}(\boldsymbol{R})-d\right| / N_{\Psi_{0}}>1$ [57].

The performance of Lanczos method depends therefore on the parameter $d$. In fact, while with the increase of the value of $d$ [within the limit defined in Eq. (10)] the measurement confidence increases (i.e., the standard deviation becomes smaller), the quality of the expectation values deteriorates (i.e., we observe a shift of the mean value). There is therefore a trade-off in the selection of $d$, which needs to be assessed independently for all observables of interest (see Fig. 10 in Appendix D).

\section{MOLECULAR DYNAMICS SCHEMES}

In this section, we present two molecular dynamics schemes for simulations in the microcanonical (isolated, NVE) and the canonical (thermalized, NVT or NPT) ensembles. In the first case, MD is performed at constant energy, and therefore a lot of care is necessary to reduce as much as possible the impact of the noise on the forces calculation.

In the second case, we will instead make use of the intrinsic noise of the quantum device to perform canonical MD simulations at constant temperature using Langevin dynamics. The tuning of the friction coefficient for the nuclear velocities will allow for the setting of the desired ensemble temperature.

\section{A. Microcanonical dynamics using Verlet integrator}

Constant energy simulations can be straightforwardly achieved by integrating Newton's equations of motion

$$
\begin{gathered}
\dot{\boldsymbol{v}}=\frac{\boldsymbol{F}(\boldsymbol{R})}{\boldsymbol{m}}, \\
\dot{\boldsymbol{R}}=\boldsymbol{v},
\end{gathered}
$$

where $\boldsymbol{v}$ is the $3 N$-dimensional vector made by the velocities of the $N$ nuclei, and $\boldsymbol{m}$ is a vector containing the masses of the $N$ particles [59]. We employ the velocity-free Verlet integrator scheme [60]

$$
\boldsymbol{R}(t+\Delta t)=2 \boldsymbol{R}(t)-\boldsymbol{R}(t-\Delta t)+\frac{\boldsymbol{F}(t)}{\boldsymbol{m}} \Delta t^{2}
$$

that updates the positions of nuclei at the next time-step $t+\Delta t$, with an error $O\left(\Delta t^{4}\right)$ and the first Verlet step given by the generic Verlet scheme $\boldsymbol{R}(t+\Delta t)=\boldsymbol{R}(t)+\boldsymbol{v}(t) \Delta t+$ $\frac{1}{2} \frac{\boldsymbol{F}(t)}{\boldsymbol{m}} \Delta t^{2}$, evaluated at $t=0$. The main issue with integrating the Newtonian equations of motion stems from the fact that (i) the systematic error prevents the nuclei to follow the exact ground state potential energy surface, and (ii) the statistical error introduces a noisy component in the forces leading eventually to instability of the dynamics. These two effects are investigated in Sec. IV.

\section{B. Generalized Langevin dynamics}

The second possibility we explore is the use of quantum forces to perform finite-temperature simulations. Langevin 
dynamics (LD) has been originally introduced in a MD context to simulate the diffusion of bodies immersed in bath of lighter particles [30]. In this framework, noisy and dissipative contributions are added to the Newton's equation of motion described above. However, LD can be employed also as a thermostat to sample from a finite-temperature canonical distribution

$$
\rho(\boldsymbol{R}, \boldsymbol{v}) \propto e^{-\beta H(\boldsymbol{R}, \boldsymbol{v})},
$$

at finite-temperature $T=1 /\left(k_{B} \beta\right)[30,61]$ with $k_{B}$ being the Boltzmann constant. We will show how the Langevin framework is particularly convenient when the forces are affected by statistical errors, as in the present setting. In this context, one can exploit the freedom given by the fluctuationdissipation theorem, which sets the relation between the friction matrix and the power spectrum of the noise. We notice that this possibility has been already put forward in the context of $a b$ initio MD [31,32]. For instance, Sorella and co-workers $[31,62,63]$ introduced this approach in quantum Monte Carlo simulations to cope with the fact that ionic forces are known only with a finite precision. This approach can be pursued both in the second order [31,63] and in the first order Langevin dynamics cases [64-66].

In this paper, we consider the second order Langevin dynamics as (i) it smoothly connects to the Newtonian equation of motion in the limit of vanishing statistical sampling error, and (ii) it has been shown that, under these conditions, some dynamical properties can be rigorously computed from thermostatted trajectories [67]. Let us start with the formal definition of generalized Langevin equation. The equations of motion read

$$
\begin{array}{r}
\dot{\boldsymbol{v}}=-\boldsymbol{\gamma}(\boldsymbol{R}) \cdot \boldsymbol{v}+\frac{\boldsymbol{F}(\boldsymbol{R})}{\boldsymbol{m}}+\eta(t), \\
\dot{\boldsymbol{R}}=\boldsymbol{v}, \quad\langle\boldsymbol{\eta}(t)\rangle=0, \\
\left\langle\eta_{i}(t) \eta_{j}\left(t^{\prime}\right)\right\rangle=\alpha_{i j}(\boldsymbol{R}) \delta\left(t-t^{\prime}\right),
\end{array}
$$

where $\eta$ is a $3 N$-dimensional vector representing the massrescaled Gaussian white noise force, with power spectrum given by $\boldsymbol{\alpha}(\boldsymbol{R})$ and $\boldsymbol{\gamma}(\boldsymbol{R})$ is a position dependent mass-rescaled friction matrix.

The fluctuation-dissipation theorem

$$
\boldsymbol{\alpha}(\boldsymbol{R})=2 T \boldsymbol{\gamma}(\boldsymbol{R})
$$

dictates the relation between the friction matrix $\boldsymbol{\gamma}(\boldsymbol{R})$ and the noise $\boldsymbol{\alpha}(\boldsymbol{R})$, in order to sample the correct finite temperature Boltzmann distribution of Eq. (14) [68,69]. The connection between the Langevin dynamics formalism and the quantum computing setting proposed in this work is established as follows. As explained in Sec. II A, each component $I \alpha$ of the forces computed with the VQE approach is subjected to the statistical error $F_{I \alpha}^{\eta}$, such that $F_{I \alpha}^{\mathrm{VQE}}=F_{I \alpha}+F_{I \alpha}^{\eta}$, where $F_{I \alpha}$ is the exact value computed with an infinite number of measurements (or in the MR). These conditions are indeed reproducing the thermal fluctuation noise that is assumed in the Langevin formalism. The connection is justified by the observation that $F_{I \alpha}^{\eta}$ is a Gaussian distributed random variable (see Appendix G). By defining $\eta_{I \alpha}=F_{I \alpha}^{\eta} / m_{I}$, we realize that the VQE process becomes compatible with the definition of the Langevin dynamics in Eq. (15). The only missing part is the friction term, which can be suitably tuned to control the system temperature. In this case, the matrix $\boldsymbol{\alpha}(\boldsymbol{R})$ is given by the covariance matrix of the forces that can be computed at each time step according to Eq. (4) as

$$
\alpha_{i j}(\boldsymbol{R})=\left\langle\left[F_{i}(\boldsymbol{R})-\left\langle F_{i}(\boldsymbol{R})\right\rangle\right]\left[F_{j}(\boldsymbol{R})-\left\langle F_{j}(\boldsymbol{R})\right\rangle\right]\right\rangle,
$$

and $\langle\cdots\rangle$ indicates the statistical average over the measurements. We notice that $\boldsymbol{\alpha}(\boldsymbol{R})$ is not constant during the dynamics as the variance of the forces is itself a stochastic quantity and varies during the simulations.

In this scheme, the value of friction is proportional to the noise fluctuations in the forces so that the corresponding nuclear displacement is also anisotropically reduced, stabilizing the dynamics while sampling unbiasedly the canonical ensemble. Finally, note that in our application the force on each atom is computed independently from all other atoms so that the matrix $\boldsymbol{\alpha}(\boldsymbol{R})$ is diagonal by construction. Despite this assumption, the diagonal elements remain a function of the atomic positions.

For the sake of demonstration we discretize Eq. (15) with the simplest Euler integrator, though much more sophisticated integration schemes exist $[31,62,63]$. We also mention that, in the most general case, an external Gaussian distributed white noise can also be added to Eq. (15), in addition to the intrinsic one present in the forces. This can be used to increase the friction, reaching an optimal value that minimizes the simulation autocorrelation time [31,62,63].

\section{RESULTS AND DISCUSSION}

To assess the quality of the force algorithm described above (see Fig. 1), we perform geometry optimization and MD studies (in the microcanonical and canonical ensembles) for two simple molecular systems: $\mathrm{H}_{2}$ and $\mathrm{H}_{3}^{+}$. The size of these molecules allows for a systematic study of the different simulation conditions while keeping the computational costs relatively low. In fact, while the quantum algorithms for electronic structure calculation show a favorable scaling comparing to the equivalent classical algorithms, their simulation with classical computers is far from efficient, especially when the classical calculation aims at reproducing the quantum variational approach, i.e., the VQE optimization as it would be implemented in a quantum computer.

To validate our approach in the case of geometry optimization and microcanonical MD simulations, we also provide a solution obtained using the matrix representation (MR) of the Hamiltonian and its direct diagonalization (Exact) to obtain the ground state energy and the corresponding eigenvector (wave function). On the other hand, to reproduce the conditions of a hardware calculation we also simulate the VQE algorithm using a realistic representation of the noise of one of the IBM Quantum processors, namely ibmq_athens. Note that MR refers to an ideal simulation where the circuit gates are represented as matrix operations that, when applied to an initial state, produce a state vector. Instead, the simulation (noisy or noiseless) of the VQE algorithm involves an emulation of the actual optimization process as it would occur on hardware. In this case, at each execution of the circuit the process returns the bit counts obtained from the measurement 


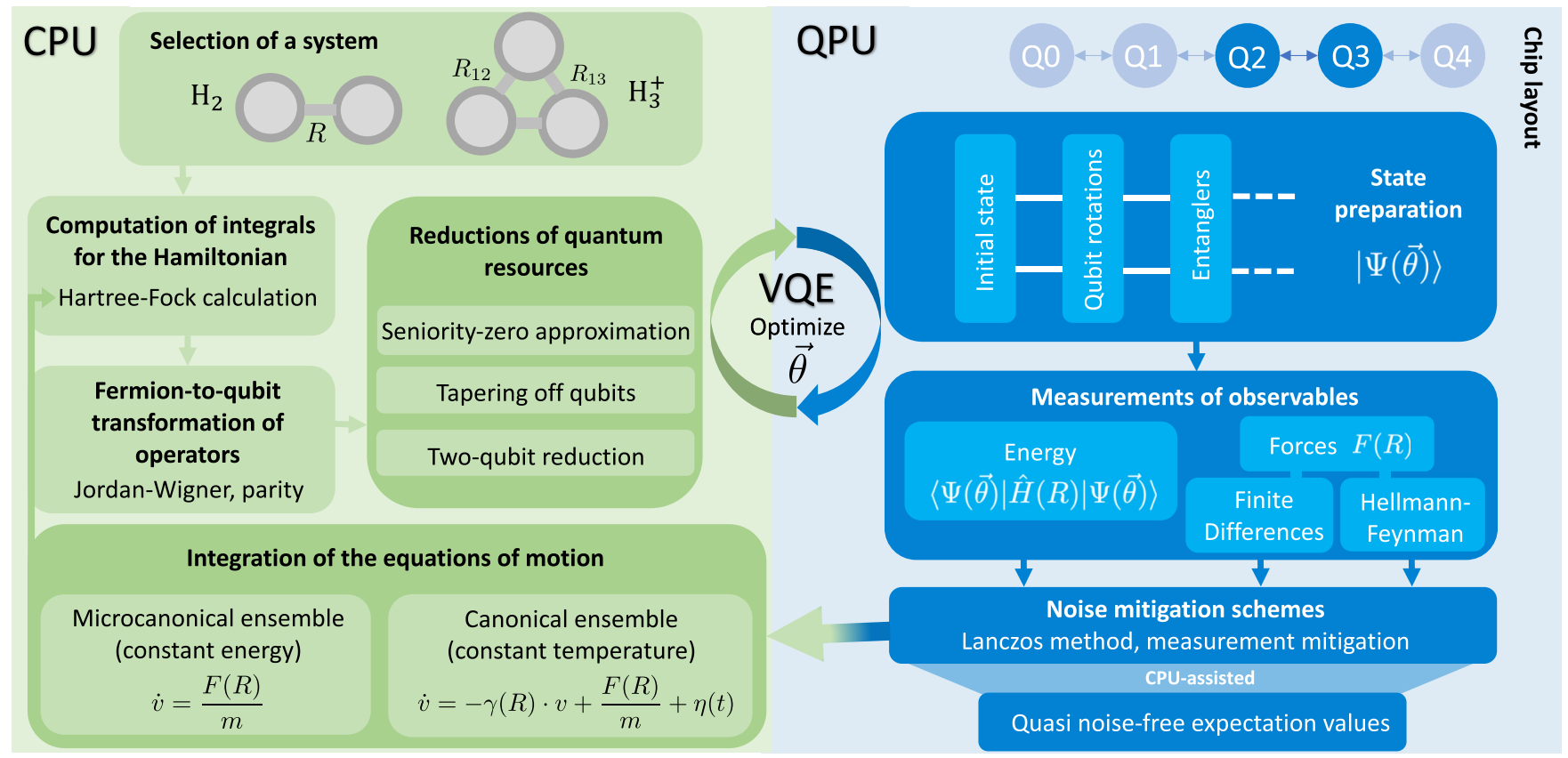

FIG. 1. Hybrid quantum-classical approach for molecular dynamics simulations in second quantization. At each time step, the classical processing unit (CPU) performs a Hartree-Fock calculation for the evaluation of the molecular Hamiltonian and of the forces operators at the given molecular geometry. The quantum processors (QPU) or a classical simulator of the QPU is then performing the optimization of the corresponding wave function (parametrized in the variables $\vec{\theta}$ ) and returns the required expectation values for the calculation of energies and forces. These values are then passed back to CPU, which performs the integration of the equations of motion in the chosen ensemble (microcanonical or canonical ensembles). On the upper right corner we show a sketch of the IBM quantum device ibmq_athens used for the hardware calculations of the $\mathrm{H}_{2}$ molecule (highlighted are the qubits used in the experiment and noisy simulations).

(collapse) of the wave function in the computational basis (see Qiskit documentation for details [56]).

Insights about the dependence of the results on the level of noise are gained by repeating the simulations with a "noise model" that corresponds to a quantum device with improved gate fidelities and halved noise rates. The details about the noise models "full" and "halved" are given in Table II of Appendix C.

For both systems we use the STO-3G basis set. A $\mathrm{HF} / \mathrm{STO}-3 \mathrm{G}$ calculation is performed to generate the molecular orbitals used to construct the Hamiltonian in second quantization. A total of four spin orbitals were used for $\mathrm{H}_{2}$ and six for $\mathrm{H}_{3}^{+}$. The number of spin orbitals is equal to the total number of qubits required for the simulations. To further reduce the computational costs, we applied qubit reductions schemes based on the symmetries of the qubit Hamiltonian. For the case of $\mathrm{H}_{2}$, we apply the "two-qubit reduction" scheme [70] reducing the final number of qubits to two. For the case of $\mathrm{H}_{3}^{+}$, we use the seniority-zero Hamiltonian (also known as doubly occupied configuration interaction Hamiltonian) $[71,72]$ which allows us to map the qubits to geminals (spatial orbitals) $[73,74]$ instead of spin orbitals reducing the number of qubits from six to three in comparison to the standard molecular Hamiltonian [see Eq. (1)]. Note that for fermionic systems the seniority is defined as the number of unpaired electrons in the Slater determinant. We further apply the "tapering off qubits" scheme [70] to reduce qubit requirements to two. A more elaborate description of these techniques and the limits of their applicability can be found in Appendix E.
The wave functions are expanded using the RY ansatz [56] with depth one, which amounts to a total of four variational parameters both for $\mathrm{H}_{2}$ and $\mathrm{H}_{3}^{+}$(see Fig. 2). The classical optimization of the VQE parameters was performed using the COBYLA optimizer [75] with default settings as defined in SciPy software library [76].

All MD calculations are performed using the HellmannFeynman formula defined in Eq. (7). The integration of the equations of motion is done using the Verlet algorithm [Eq. (13)] and a time step of 0.2 fs for microcanonical MD and the Langevin algorithm (Eq. (15)).

For the Lanczos algorithm (see Eq. (8)), we use $d_{\mathrm{PES}}=$ -0.4 for the evaluation of the potential energy and $d_{\text {forces }}=$ -0.1 for the forces. The noise models used in the simulations are summarized in Appendix $\mathrm{C}$, while the implemented measurement (readout) error mitigation scheme is described in the documentation of Qiskit [56].

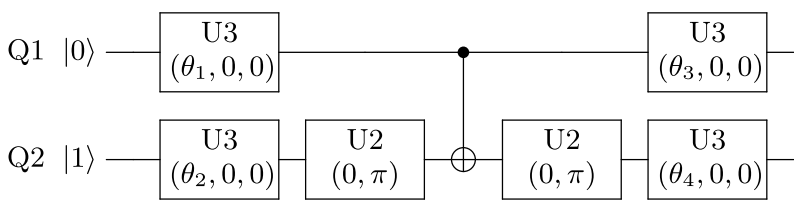

FIG. 2. Quantum circuit corresponding to the RY ansatz. The VQE variational parameters $\vec{\theta}=\left(\theta_{1}, \theta_{2}, \theta_{3}, \theta_{4}\right)$ control the rotations on the Bloch sphere with $U 3(\theta, \phi, \lambda)=R_{z}(\phi-$ $\pi / 2) R_{x}(\pi / 2) R_{z}(\pi-\theta) R_{x}(\pi / 2) R_{z}(\lambda-\pi / 2)$ and $U 2(\phi, \lambda)=$ $R_{z}(\phi+\pi / 2) R_{x}\left(\frac{\pi}{2}\right) R_{z}(\lambda-\pi / 2)$. In particular, $U 3(\theta, 0,0)=R_{y}(\theta)$ hence the name of the ansatz. For further details see Appendix F. 


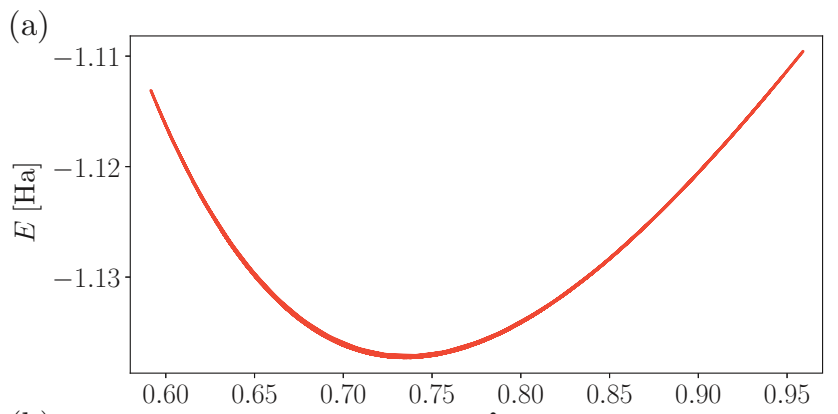

(b)

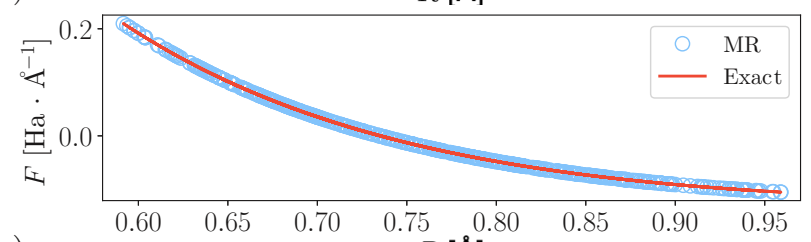

(c)

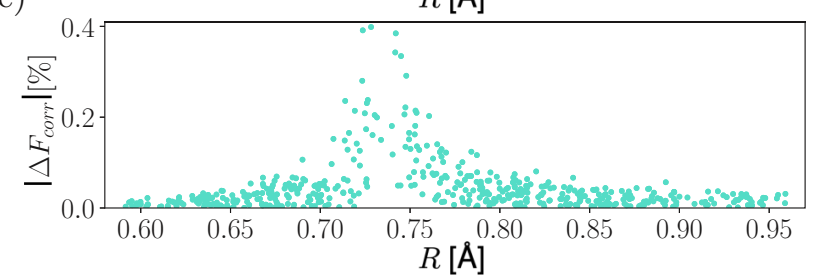

FIG. 3. (a) Potential energy obtained by exact diagonalization of the $\mathrm{H}_{2}$ Hamiltonian. (b) Forces along the molecular axes calculated with finite differences on the curve in (a) (denoted by Exact) and with the H-F approach (see Eq. (7), denoted by MR). A displacement $\Delta R=10^{-3} \AA$ is used in both cases. (c) Percent error on the forces due to the omission of the Pulay component.

In the case of $\mathrm{H}_{2}$, we also performed a short (20 fs long) hardware microcanonical MD calculation using the IBM chip $i b m q \_a t h e n s$. The characterization of this device are given in Appendix C.

\section{A. Validation of the H-F forces calculation}

To verify the quality of the H-F forces and estimate the error done by neglecting the last two components in Eq. (5) (Pulay's forces), we performed an exact calculation of the forces for $\mathrm{H}_{2}$ by means of the direct differentiation (using finite differences with a displacement of $10^{-3} \AA$, see Eq. (6)) of the potential energy surface obtained by exact diagonalization of the system Hamiltonian (Fig. 3(a)). These noiseless forces are then compared to the H-F ones computed with Eq. (7) (Fig. 3(b)), while the absolute percental error computed as $\left|\Delta \boldsymbol{F}_{\text {corr }}\right|=\left|\boldsymbol{F}_{\text {exact }}-\boldsymbol{F}_{\text {MR }}\right| /\left|\boldsymbol{F}_{\text {MR }}\right|$ is reported in Fig. 3(c). The maximal deviation is observed for geometries close to the equilibrium bond distance and never exceeds $0.4 \%$ of the total force. This is probably due to numerical errors arising from the addition of small values of opposite sign. Since the error due to statistical sampling as well as the hardware noise are much larger than this value, we can safely neglect the Pulay contributions for the remainder of this work.

\section{B. Geometry optimization of $\mathrm{H}_{2}$ and $\mathrm{H}_{3}^{+}$: Simulations}

In this section, we use the most accurate force calculation setup, i.e., Eq. (7) with Lanczos noise mitigation, to evaluate
TABLE I. Geometry optimization results using the MR (reference), the VQE, and the VQE with Lanczos (VQE-L) algorithms. In the two last cases, we use 8192 measurements for the evaluation of the energy and force components. The equilibrium bond distance of $\mathrm{H}_{2}$ is given by $R_{\text {eq. }}$. The structure of $\mathrm{H}_{3}^{+}$(see Fig. 1) is characterized by three parameters: (i) the distance between atoms 1 and $2\left(R_{12}\right)$, (ii) the distance between atoms 1 and $3\left(R_{13}\right)$, and (iii) the angle $\alpha_{213}$ formed between the bonds 1-2, and 1-3 $\left(\alpha_{213}\right)$. We use A for distances and degrees for angles.

\begin{tabular}{lcccc}
\hline \hline & & MR & VQE & VQE-L \\
\hline $\mathrm{H}_{2}$ & $R_{\mathrm{eq}}$ & 0.735 & 0.742 & 0.733 \\
$\mathrm{H}_{3}^{+}$ & $R_{12}$ & 0.985 & 1.006 & 0.990 \\
& $R_{13}$ & 0.985 & 0.999 & 0.990 \\
& $\alpha_{213}$ & 60.0 & 59.8 & 59.9 \\
\hline \hline
\end{tabular}

the optimized geometry for $\mathrm{H}_{2}$ and $\mathrm{H}_{3}^{+}$using a realistic model of the $i b m q \_a t h e n s$ device. The results are summarized in Table I.

We observe that the forces evaluated with the standard VQE using the noise model of a real device (ibmq_athens) have errors in the order of 0.01-0.02 $\AA$ in the bond distances and $0.2^{\circ}$ in the angle $\alpha_{213}$ (see Fig. 1). Our results for $\mathrm{H}_{2}$ are in agreement with the work of O'Brien et al. [28] where, using Hellmann-Feynman gradients in combination with the Newton's optimization method, they obtained a similar error of $0.014 \AA$ for the equilibrium bond length (for the same STO-3G basis set). Note that in Ref. [28] the optimization was performed using an approximate molecular Hessian matrix, which, however, is not necessary for the simple applications investigated in this work. By using the Lanczos mitigation scheme, we improve significantly the quality of the forces and, consequently, the quality of the optimized structures (i.e., reducing the error on bond distances to 0.002-0.005 $\AA$ ). As it will become evident in the study of the MD, even though the error on the energies are still not within the so-called chemical accuracy (1 kcal/mol or $1.6 \mathrm{mHa})$, the forces computed directly using Eq. (7) show a great level of accuracy (see Fig. 5) and can be used to compute optimized molecular geometries.

\section{Microcanonical dynamics of $\mathrm{H}_{2}$ : Simulations}

In this section, we apply the forces derived in Eq. (7) for the calculation of microcanonical MD trajectories of $\mathrm{H}_{2}$.

In the first case $\left(\mathrm{H}_{2}\right)$ we perform a systematic study of the effect of the different noise sources (hardware and statistical) on the quality of the dynamics. All calculations of this section are performed using the noise models (for depolarization, thermalization, and readout errors) reported in Appendix C, parametrized using the calibration data of the ibmq_athens device (see Table II, in Appendix C). To shed light on the potential future improvements associated with the development of future hardware, we also present results for the half of the current hardware noise. In particular, we present the time series of kinetic, potential, and total energies for three different simulations conditions: (i) VQE with 8192 measurements for the determination of the expectation values of energies and forces; (ii) VQE with 81920 measurements for an improved accuracy of both energy and force estimations due to lowering 

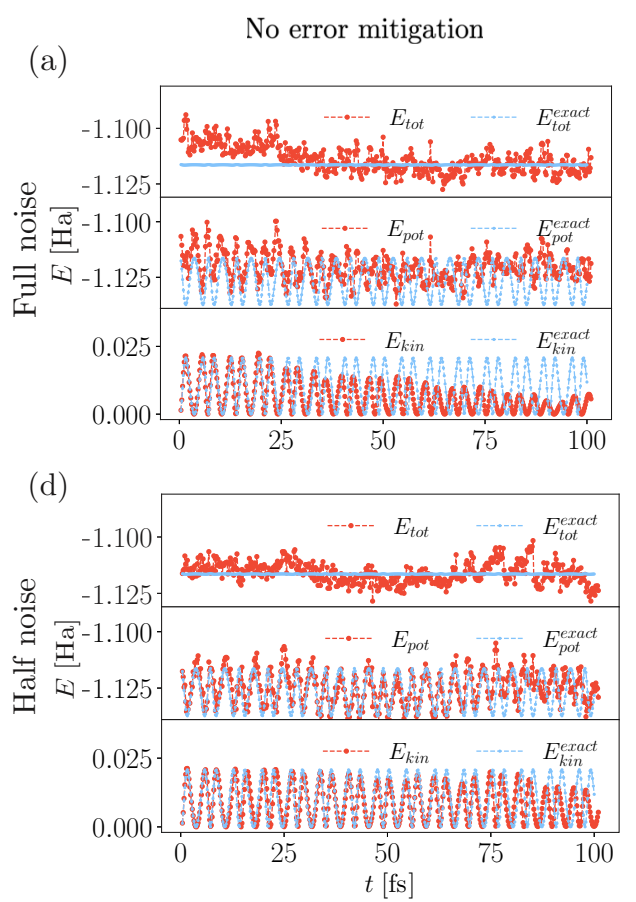

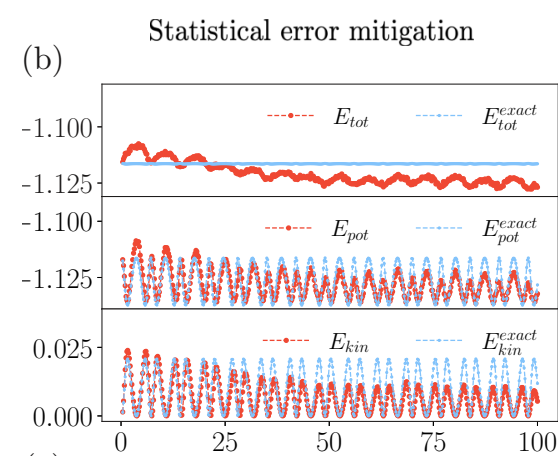

(e)

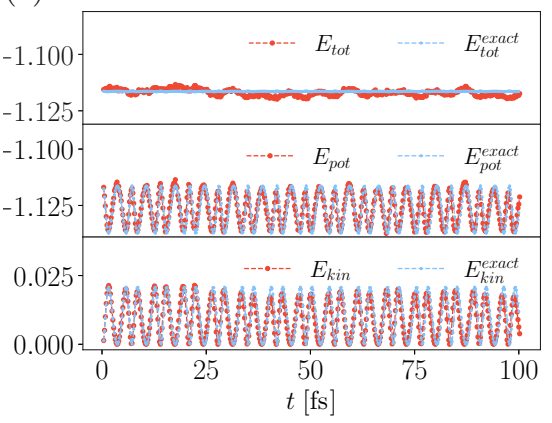

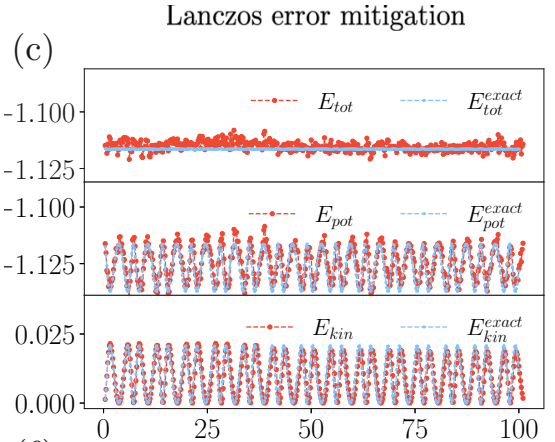

(f)

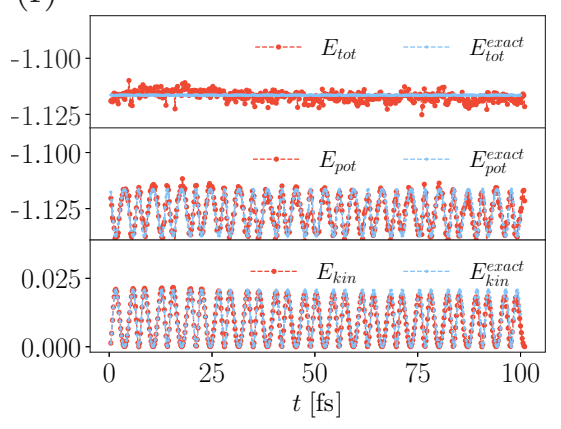

FIG. 4. Time series of the total, potential, and kinetic energies for the dynamics of the $\mathrm{H}_{2}$ molecule $(t=100 \mathrm{fs}, d t=0.2 \mathrm{fs})$ using the VQE algorithm with the realistic noise corresponding to the $i b m q \_a t h e n s$ device and: (a) 8192 measurements for the evaluation of energies and forces, (b) 81920 measurements for the evaluation of energies and forces, and (c) the VQE-L error mitigation scheme with 8192 measurements for the evaluation of energies and forces. (d), (e), and (f) The same simulations as in (a), (b), and (c) with halved hardware noise level. In all panels, the blue curves correspond to the reference "exact" dynamics obtained in the MR.

of the statistical noise; and (iii) VQE with 8192 measurements combined with the Lanczos noise mitigation algorithm with $d_{\mathrm{PES}}=-0.4$ and $d_{\text {forces }}=-0.1$ (see Eq. (8)). Note that the choice of 8192 measurements for a single experiment is imposed by the provider of the IBM quantum hardware and is not an intrinsic limitation of the devices. In all cases, we will take the microcanonical MR trajectory as reference, exact dynamics of the system. The microcanonical dynamics were performed with the Verlet algorithm described in Sec. III A and using an initial bond length of $0.6 \AA$. The results of these three simulations are reported in Figs. 4(a), 4(b), and 4(c), respectively.

In all cases, we observe that in all $\mathrm{VQE}$ noisy simulations the accuracy of the kinetic energy is much higher than the one for the potential energy. Here and in the following we use the adjective noisy to emphasize the use of noise model in the classical simulations of the VQE algorithm. This is possible because the forces are computed independently from the energy using the expectation values in Eq. (7). As a result, we obtain reliable dynamics even when the overall error in the potential energy is higher than what would be required for numerical differentiation. In the case of the bare VQE simulation (Figs. 4(a) and 4(d)), the full noise dynamics reproduces quite accurately the vibrational frequency of the bond oscillation even though an important damping is also observed, which is not compensated by an equal increase in the potential energy. As a consequence the total energy is not conserved (Fig. 4(a)). We can therefore interpret the noise as an external bath that modulates the total energy of the $\mathrm{H}_{2}$ subsystem. We will fully exploit this prop- erty in the Langevin dynamics reported in the next section (Sec. IV F). When halving the level of noise (Fig. 4(d)), we observe an important improvement of the total energy conservation and a more accurate oscillation frequency compared to the reference, noiseless, MR calculation. This indicates that the hardware noise is currently among the most important limitations for microcanonical dynamics, even though with the superconducting qubit technology we are not far from reaching the accuracy necessary for simulations in the picosecond timescale (see also the results of the hardware MD calculation of $\mathrm{H}_{2}$ in Fig. 6 of Sec. IV D).

In Figs. 4(b) and 4(e) we present the case in which we increase the sampling statistics for the calculation of the energy and force expectation values from 8192 to 81920 . As expected, we observe a general decrease of the overall scattering of the energy values due to the improved statistics. However, the same issue with the conservation of total energy observed in Figs. 4(b) and 4(e) persists in both cases, full and halved noise levels, even though to a much lesser extent in the last case. Once more, this corroborates the hypothesis that the hardware noise (and not the statistic sampling) is the main source for the nonconservation of the total energy.

Finally, in Figs. 4(c) and 4(f) we report the simulation using the Lanczos noise mitigation scheme (VQE-L). For both levels of noise, the reproduction of the reference MR dynamics is extremely good, despite the use of the minimal sampling statistics, i.e., 8192 measurements for each expectation value. The conservation of total energy is maintained over the total simulation length and the bond frequency is captured very closely. 


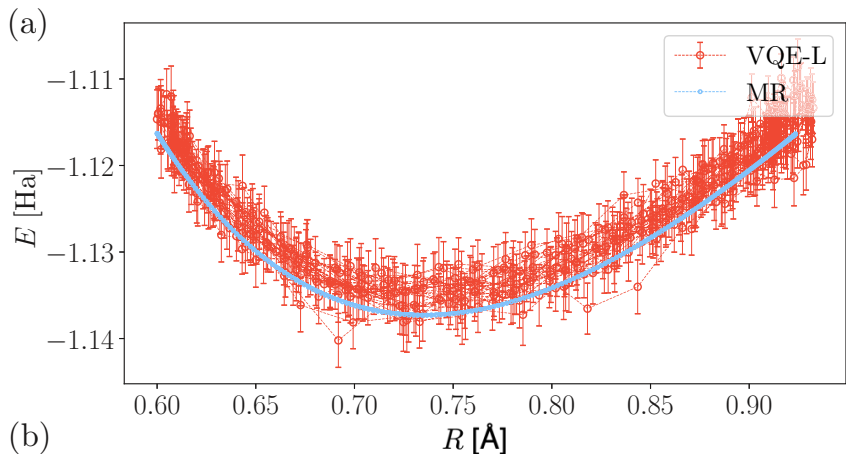

(b)

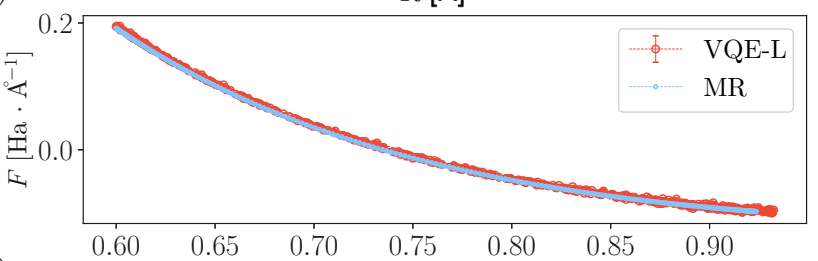

(c)

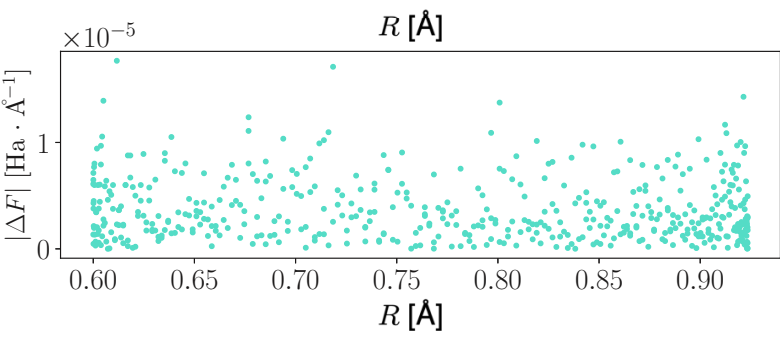

FIG. 5. (a) Sampling of the potential energy obtained with the $100 \mathrm{fs}$ VQE-L dynamics of $\mathrm{H}_{2}$ shown in Fig. 4(e). The blue line corresponds to the MR result. (b) Corresponding forces along the molecular axes acting on one of the hydrogen atom as a function of the bond distance. (c) Norm of the difference between the forces computed with VQE-L and MR. MR forces evaluated at the same geometries sampled along the VQE-L trajectory.

We note that the same level of accuracy obtained with the Lanczos algorithm (Fig. 4(f)) is also achieved in simulations with halved noise level and improved read out statistics (Fig. 4(e)). This bodes well for the use of the proposed MD algorithm in near-term quantum devices, which in the future can be made more efficient and accurate without the need of applying computationally expensive error mitigation schemes.

In Fig. 5 we give an overview on the performance of the VQE-L algorithm for microcanonical MD by reporting each energy value of the structures sampled along the trajectory in Fig. 4(c) as a function of the corresponding bond length. Once more, we notice that even though the error in the potential energy is of the order of several mHa, using Eq. (7) we can obtain fairly reliable forces (see Fig. 5(b)), which can be used for accurate geometry optimizations and MD simulations. The components of the forces acting on one of the hydrogen atoms along the molecular axes are shown in Fig. 5(b) together with the reference $\mathrm{H}-\mathrm{F}$ forces obtained with the MR approach. Finally, in Fig. 5(c) we report the difference between the $\mathrm{H}-\mathrm{F}$ forces computed with VQE-L and the ones obtained with the reference MR approach. We notice that, for this particular example, the magnitude of the error on the forces is approximately equally spread over the entire bond range with a magnitude smaller than $2.0 \times 10^{-5} \mathrm{Ha} \AA^{-1}$, which is

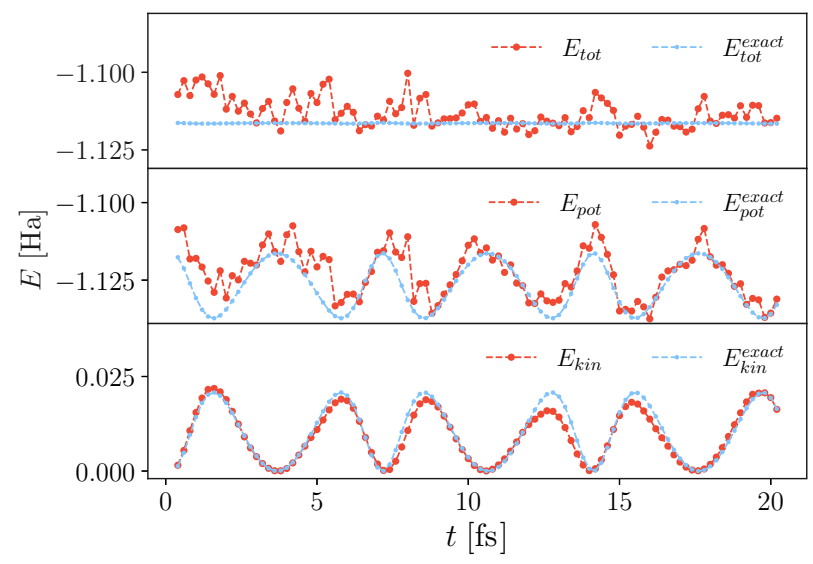

FIG. 6. Time series of the total, potential, and kinetic energies for the dynamics of the $\mathrm{H}_{2}$ molecule on the IBM quantum computer $i b m q \_a t h e n s$. Calculations were done using the bare VQE algorithm (without Lanczos noise mitigation) with 8192 measurements per expectation value. The equation of motion was solved with the velocity-free Verlet algorithm and a time step of $0.2 \mathrm{fs}$.

already a fairly good accuracy considering the level of noise in the quantum processors.

\section{Microcanonical dynamics of $\mathrm{H}_{2}$ : Hardware calculations}

As a final demonstration of the accuracy of the proposed algorithm, we perform constant energy dynamics of $\mathrm{H}_{2}$, where forces and energies are calculated on the IBM quantum computer ibmq_athens (see Fig. 1). The same approach could be also applied to the Langevin dynamics; however, due to the long equilibration times this would require a large number of steps that we cannot afford yet. The characteristic features of ibmq_athens at the time of execution (13 July 2020) are summarized in Table II, Appendix C.

The dynamics on hardware obtained with the bare VQE algorithm (without Lanczos noise mitigation) and the velocityfree Verlet integration scheme with a time step of 0.2 fs confirms the quality of the noise models used in the simulations reported in Sec. IV C (see Fig. 6). Once more, we observe that despite the quite large deviations in the potential energy (of about 10-15 $\mathrm{mHa}$ using the canonical 8192 measurements) the accuracy of the kinetic energy is an order of magnitude larger. This is due to the stability of the force calculation using the expectation values introduced in Eq. (7), which bypasses the derivative of the noisy potential energy surface.

The stability of this microcanonical dynamics scheme is such that we do not need to apply the Lanczos noise mitigation scheme, which will require the evaluation of additional expectation values, with an impact on the overall machine time.

\section{E. Microcanonical dynamics of $\mathbf{H}_{3}^{+}$: Simulations}

The $\mathrm{H}_{3}^{+}$molecule is an interesting system for the validation of our MD algorithm. While conserving the same number of electrons as in $\mathrm{H}_{2}$, the size of the configuration space increases to 9 (6 if we remove the translational degrees of freedom) introducing additional dynamical degrees of freedom, such as collective bond vibrations. Building from 


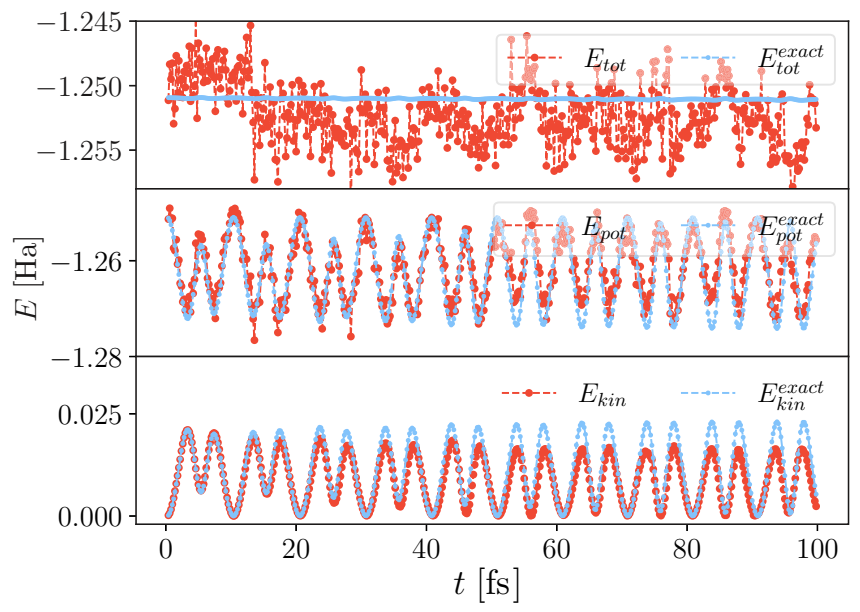

FIG. 7. Evolution of the total, potential, and kinetic energies for the dynamics of the $\mathrm{H}_{3}^{+}$molecule $(t=100 \mathrm{fs}, d t=0.2 \mathrm{fs}$ ) using the VQE-L algorithm with realistic noise of $i b m q$ athens chip and 8192 shots (fixed $d$ method, $d_{\mathrm{PES}}=-0.4$ and $d_{\text {Forces }}=-0.1$ ). Details of the calculation are described in Sec. IV.

(a)
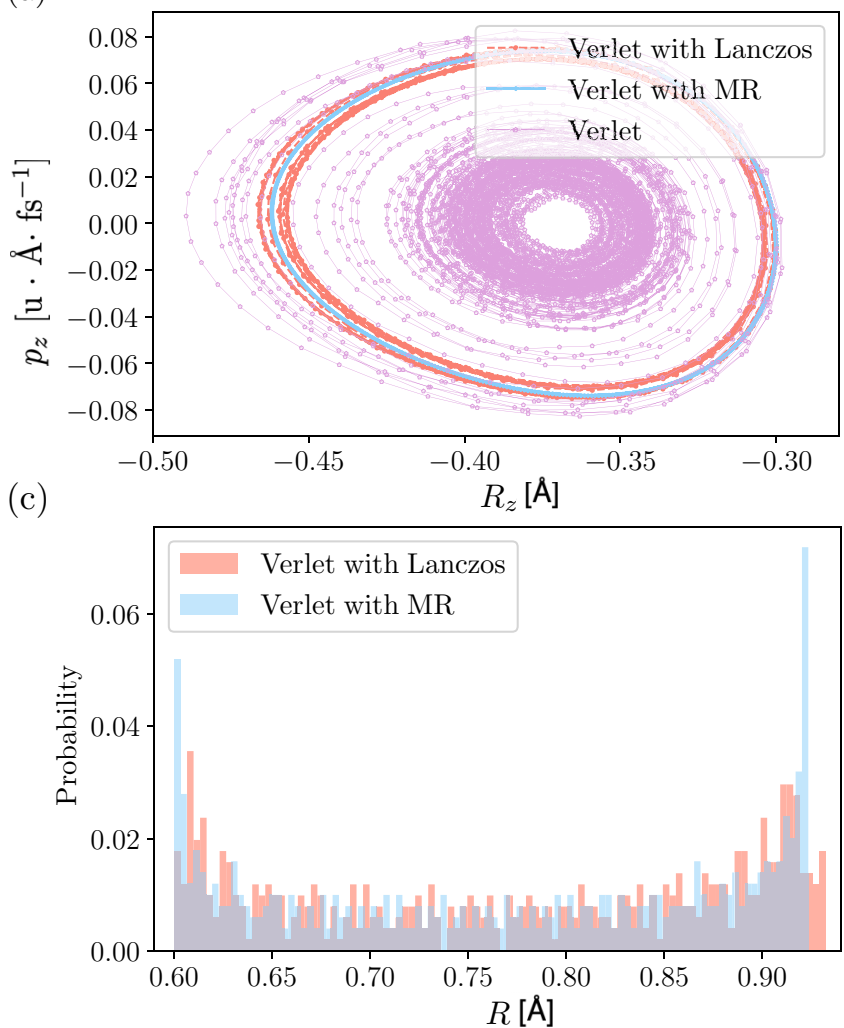

what we learned in the previous section, we will restrict the study of the microcanonical dynamics of $\mathrm{H}_{3}^{+}$to the use of the VQE-L algorithm with the velocity-free Verlet for the integration of the equations of motion. As initial conditions we take an off-equilibrium geometry characterized by the internal parameters $d_{12}=1.245 \AA, d_{13}=1.245 \AA$, and $\alpha_{213}=48.5^{\circ}$ (see Table I for the corresponding equilibrium values) and zero velocities for all atoms. The time series for the kinetic, potential, and total energies obtained over $100 \mathrm{fs}$ of NVE dynamics are given in Fig. 7. As for the case of $\mathrm{H}_{2}$, we observe a fairly good energy conservation with a drift of about $5 \mathrm{mHa}$ over $100 \mathrm{fs}$ of dynamics. Also important is the accuracy with which the dynamics can capture the nontrivial molecular oscillations, as illustrated by the evolution of the kinetic energy (bottom panel in Fig. 7) and the agreement with the reference MR calculations.

\section{F. Langevin dynamics of $\mathrm{H}_{\mathbf{2}}$ : Simulations}

In this section, we demonstrate the Langevin dynamics driven by the statistical noise on the evaluation of the forces

(b)

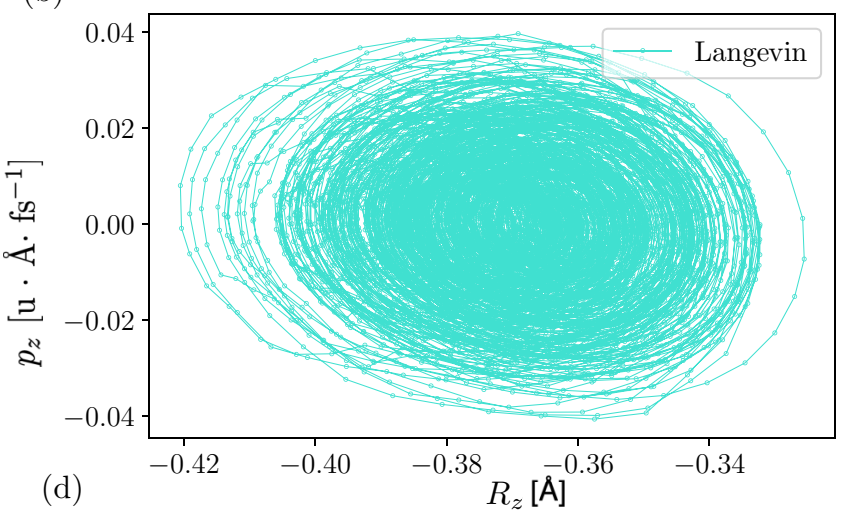

(d)

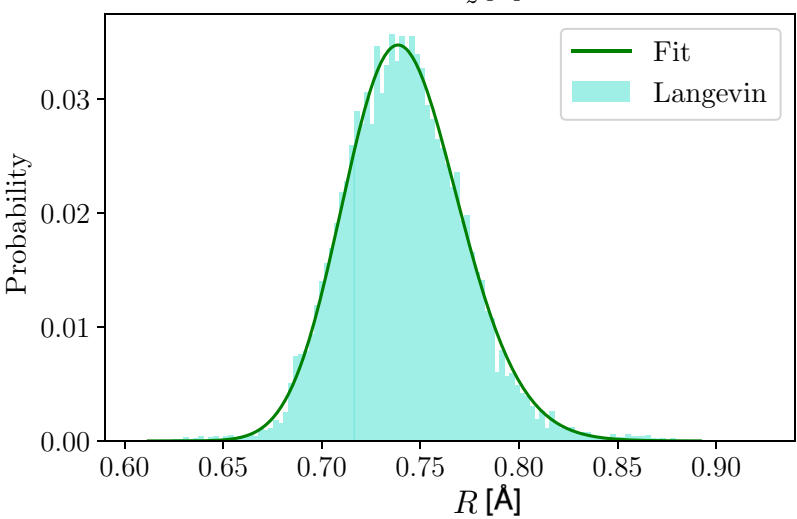

FIG. 8. Phase space orbits corresponding to one of the hydrogen atoms of $\mathrm{H}_{2}$ : (a) Microcanonical dynamics with MR, VQE, VQE-L. (b) Constant temperature Langevin dynamics at an average temperature $T=422 \mathrm{~K}$. Distribution of the bond length from the simulations with: (c) Verlet (initial bond length $R=0.6 \AA, 8192$ shots) and (d) Langevin dynamics (initial bond length $R=0.73 \AA$, 1024 shots). In the microcanonical case, the noisy and noiseless simulations are denoted with Lanczos and MR, respectively. The parameters for the Lanczos mitigation are described in Sec. IID. The Langevin canonical bond distribution (panel (d)) is fitted to the Boltzmann distribution using a Morse potential $\rho(R) \propto e^{-\beta\left[c\left(e^{-2 m\left(R-r_{\mathrm{eq}}\right)}-2 e^{-m\left(R-r_{\mathrm{eq}}\right)}\right)\right]}$, with the following parameter $\beta=748 \mathrm{Ha}^{-1}$. The values of $c=0.106 \mathrm{Ha}, m=2.725 \AA^{-1}$, $r_{\mathrm{eq}}=0.739 \AA$ were obtained from a separate fit of the PES computed with the MR approach. 
(see Secs. II A and III B) on $\mathrm{H}_{2}$. To this end, we observe that Eq. (15) defines a stochastic process having as fixed point the unique equilibrium distribution given in Eq. (14). In Fig. 8(d) we plot the distribution of the bond lengths $R$ sampled via a Langevin dynamics of total length $t=2.8$ ps using 1024 shots and $d t=0.2 \mathrm{fs}$, resulting in a measured temperature of $T_{\text {kin }}=420 \pm 100 \mathrm{~K}$ (from the expectation values of the kinetic energy). Statistical error bars on the measured temperature are obtained with the standard binning technique [68] and can be decreased by running longer simulations. By contrast, the number of shots used to compute the expectation values controls the friction value, hence the autocorrelation time of the sampling.

The normalized distribution $\rho(R)$ that we obtain, with $R$ being the bond length, can be fitted by the canonical distribution Eq. (14) (marginalized over the relative momentum $\left.p_{z}\right), \exp [-\beta U(R)]$, where $U(R)$ is the Morse potential fit of the MR potential energy surface of $\mathrm{H}_{2}$, and $T=1 /\left(k_{B} \beta\right)=$ $422 \pm 30 \mathrm{~K}$ is a fitted temperature (errors estimated as 3 standard deviations). The agreement between $T$ and $T_{\text {kin }}$ shows that a finite-temperature distribution can be achieved by means of the Langevin approach.

On the other hand, it is qualitatively evident that the distribution of bond lengths produced by the noisy Verlet dynamics (see Fig. 8(c)) is not in agreement either with a Boltzmann distribution, which is peaked around $R_{\mathrm{eq}}$, or with the microcanonical one, which is sharply peaked at the turning points of the trajectory.

The difference in the sampling between the Langevin and Verlet integrator is also shown in Fig. 8. Panel (a) depicts the phase space orbits of one of the two hydrogen atoms of the $\mathrm{H}_{2}$ molecule for different microcanonical simulations. The exact dynamics obtained in the MR representation of the quantum circuit (in blue) shows the well-defined orbit typical of a constant energy MD. The trajectory obtained with the VQE-L algorithm (in orange) is covering a thin annulus in the phase space that closely follows the exact orbit. This behavior can be associated with the small total energy fluctuations observed in Fig. 4(c), which originate from the hardware noise. By removing the noise mitigation correction (violet curve), the microcanonical dynamics obtained with the Verlet algorithm under the influence of the hardware and statistical noises produces a dynamics that resembles a constant temperature MD leading to the sampling of a thick annulus in phase space. Since in the first $100 \mathrm{fs}$ of the bare VQE dynamics shows a drop of the total energy (Fig. 4(a)), the orbit is mainly sampling the low energy region of the phase space. As expected, all microcanonical MD trajectories sample a bimodal distribution of the molecular bond length with two maxima at the turning points (Fig. 8(c)). Finally, in Fig. 8(b) we report an equivalent phase space trajectory for the canonical Langevin dynamics of $\mathrm{H}_{2}$. Due to the different choice of the initial bond length, the trajectory is sampling a different portion of the phase space while the coupling to the noise is keeping the system at an average temperature of about $422 \mathrm{~K}$. Most importantly, the Langevin dynamics reproduces the correct Gaussian-like canonical distribution of the bond length (Fig. 8(d)).

\section{CONCLUSION}

In this work we implemented a quantum algorithm for the calculation of accurate forces within the VQE framework. To this end, we used the ground state wave function to directly measure the expectation values of the force operator associated with each atom of the system. In this way, we obtain high quality Hellmann-Feynman forces, which show larger robustness against hardware and readout noises than the energies. We demonstrated that the quality of the forces is not affected by the omission of the components arising from the direct derivative of the ground state wave function (i.e., the Pulay components).

In addition, by applying the Lanczos mitigation scheme on the forces evaluation we are able to achieve an accuracy that allows for the calculation of optimized molecular geometries with errors of about $0.005 \AA$ for the bond lengths and of $0.1^{\circ}$ for the angles in two test cases: the $\mathrm{H}_{2}$ and $\mathrm{H}_{3}^{+}$molecules. Using the same algorithm, we have also performed molecular dynamics simulation of $\mathrm{H}_{2}$ and $\mathrm{H}_{3}^{+}$in the constant energy (microcanonical) ensemble as well as in the constant temperature (canonical) ensemble using the Langevin approach. All calculations were performed using a realistic description of the hardware and readout errors corresponding to state-ofthe-art IBM quantum computers. Despite the sizable errors in the evaluation of the potential energy, the direct calculation of the forces enables the evaluation of accurate trajectories as demonstrated by the accuracy of the kinetic energy profiles, which is in good agreement with the reference calculations. Also in this case, the use of the Lanczos mitigation scheme in VQE (VQE-L) gives rise to a significant improvement of the dynamics, as can be seen from the conservation of the total energy of the system. We also stress the fact that by increasing the number of sampling points (readout measurements) or halving the level of noise we obtain a noticeable improvement on the quality of the simulations. In particular, the VQE-L approach with full hardware noise provides forces with an accuracy of about $10^{-5} \mathrm{Ha} \AA^{-1}$. Motivated by the promising results obtained with the simulations, we also performed $20 \mathrm{fs}$ of dynamics of $\mathrm{H}_{2}$ on the ibmq_athens device obtaining stable and accurate description of the molecular vibration.

Finally, we investigated the possibility to take advantage of the statistical noise inherent to the measurement process of the expectation values of the forces to perform constant temperature MD using the Langevin algorithm. In particular, we showed that by a suitable choice of measurement noise level through the number of shots we can control the autocorrelation time of the simulations and accurately sample the Boltzmann distribution for the $\mathrm{H}_{2}$ molecule at a given temperature. However, further investigations are necessary to improve the coupling to the reservoir (i.e., computation of off-diagonal elements in the force covariance matrix) and the corresponding selection of an optimal friction coefficient through a better refined coupling scheme.

Concerning the scaling, even though the number of qubits and the infidelities of the gate operations are limiting the size of the systems that can be investigated with near-term quantum computers, the use of embedding schemes, such as the one described in Ref. [77], will soon make possible 
the calculation of the dynamics of relatively complex molecular systems. In addition, the calculation of forces within the $\mathrm{H}-\mathrm{F}$ framework requires the same computational cost as for the evaluation of the ground state wave function using the undisplaced Hamiltonian; there is no need for additional measurements (of Pauli strings) than the ones used in the VQE for the determination of the ground state energy. The only difference occurring in the calculation of forces lies in the coefficients (i.e., the classical integrals) associated with Pauli strings, which are based on integrals computed at the displaced geometries (characterized by the $\hat{H}_{+}$and $\hat{H}_{-}$Hamiltonians). For these reasons, we believe that the quantum MD algorithm proposed in this work will effectively become an interesting application for near-term quantum computers.

We can therefore conclude that, despite the sizable error in the evaluation of the ground state energy of molecular system, the quality of the forces evaluated using the VQE algorithm, including error mitigation schemes such as the proposed Lanczos approach, enables the calculation of accurate optimized geometries as well as stable constant energy and constant temperature MD trajectories, opening up new avenues for the use of quantum computers in molecular chemistry and physics.

\section{ACKNOWLEDGMENTS}

The authors acknowledge the financial support from the Swiss National Science Foundation (SNF) through Grant No. 200021-179312. We also thank Prof. Jürg Hutter for insightful discussions. IBM, the IBM logo, and ibm.com are trademarks of International Business Machines Corp., registered in many jurisdictions worldwide. Other product and service names might be trademarks of IBM or other companies. The current list of IBM trademarks is available at [78].

\section{APPENDIX A: ONE-/TWO-ELECTRON INTEGRALS}

The coefficients of the one-/two-body excitations in Eq. (1) are given, respectively, by one-/two-electron integrals in physics notation

$$
h_{r s}(\boldsymbol{R})=\int d \boldsymbol{r}_{1} \phi_{r}^{*}\left(\boldsymbol{r}_{1}\right)\left(-\frac{1}{2} \nabla_{\boldsymbol{r}_{1}}^{2}-\sum_{I=1}^{M} \frac{Z_{I}}{R_{1 I}}\right) \phi_{s}\left(\boldsymbol{r}_{1}\right),
$$

and likewise for the two-electron terms

$$
g_{p q r s}(\boldsymbol{R})=\int d \boldsymbol{r}_{1} d \boldsymbol{r}_{2} \phi_{p}^{*}\left(\boldsymbol{r}_{1}\right) \phi_{q}^{*}\left(\boldsymbol{r}_{2}\right) \frac{1}{r_{12}} \phi_{r}\left(\boldsymbol{r}_{2}\right) \phi_{s}\left(\boldsymbol{r}_{1}\right),
$$

where $Z_{I}$ is the nuclear charge of atom $I, R_{1 I}=\left|\boldsymbol{R}_{I}-\boldsymbol{r}_{1}\right|$ is the distance between the nuclei $I$ and electron 1 , and $r_{12}=\mid \boldsymbol{r}_{1}$ $\boldsymbol{r}_{2} \mid$ is the distance between two electrons at positions $\boldsymbol{r}_{\mathbf{1}}$ and $\boldsymbol{r}_{\mathbf{2}}$. In this work, the computation of elements defined Eqs. (A1) and (A2) is performed using a HF calculation with PySCF software [79].

\section{APPENDIX B: PAULI MATRICES}

We use the following notation for the Pauli matrices:

$$
\begin{gathered}
I \equiv \mathbb{I}=\left(\begin{array}{ll}
1 & 0 \\
0 & 1
\end{array}\right), \quad X \equiv \sigma^{x}=\left(\begin{array}{ll}
0 & 1 \\
1 & 0
\end{array}\right), \\
Y \equiv \sigma^{y}=\left(\begin{array}{cc}
0 & -i \\
i & 0
\end{array}\right), \quad Z \equiv \sigma^{z}=\left(\begin{array}{cc}
1 & 0 \\
0 & -1
\end{array}\right) .
\end{gathered}
$$

\section{APPENDIX C: ANALYSIS OF THE NOISE SOURCES}

In Table II, we provide the noise model used in our simulations. The given information (T1, T2, qubit frequencies, readout errors, error rates for single qubit, and two-qubit gates per qubit) will enable the reconstruction of our calculations in Qiskit [56].

The considered error sources are the depolarization, thermalization, and readout errors. Next we present a brief summary of how they are modeled in Qiskit:

(i) The depolarization error consists of driving the noiseless density matrix $\hat{\rho}=|\Psi\rangle\langle\Psi|$, with $|\Psi\rangle$ being a pure state, to the general uncorrelated density matrix $1 / 2^{N_{q}}$ as

$$
\hat{\rho}_{d}=\gamma_{1} \operatorname{Tr}[\hat{\rho}] \mathbf{1} / 2^{N_{q}}+\left(1-\gamma_{1}\right) \hat{\rho},
$$

with $N_{q}$ being the number of qubits and $\gamma_{1}$ representing the decay to the uncorrelated state. The decay is estimated using gate fidelities given in Table II.

(ii) The thermalization error (e.g., general amplitude dampening and phase flip error) of a qubit can be written as decay towards the Fermi-Dirac distribution of ground and excited states based on their energy difference $\omega$ :

$$
\hat{\rho}_{t}=p|0\rangle\langle 0|+(1-p)| 1\rangle\langle 1|,
$$

with $p=\left(e^{\frac{-\omega}{k_{b} T}}+1\right)^{-1}, T$ being the temperature and $k_{B}$ the Boltzmann constant.

(iii) The readout error is classically modeled by calibrating the so-called measurement error matrix that assigns to any $N_{q}$-qubit computational basis state $|i\rangle$ (i.e., correct state that should be obtained) a probability to readout all the states

\begin{tabular}{|c|c|c|c|c|c|c|}
\hline Qubit & $\mathrm{T} 1(\mu \mathrm{s})$ & $\mathrm{T} 2(\mu \mathrm{s})$ & Freq. (GHz) & Readout error & Single-qubit U2 error rate & CNOT error rate \\
\hline Q0 & 64.915 & 104.232 & 5.176 & $1.000 \times 10^{-2}$ & $2.586 \times 10^{-4}$ & cx0_1: $7.982 \times 10^{-3}$ \\
\hline Q1 & 62.179 & 73.999 & 5.267 & $2.000 \times 10^{-2}$ & $3.186 \times 10^{-4}$ & cx1_0: $7.982 \times 10^{-3}, \mathrm{cx} 1 \_2: 8.136 \times 10^{-3}$ \\
\hline Q2 & 83.292 & 100.716 & 5.052 & $2.333 \times 10^{-2}$ & $3.440 \times 10^{-4}$ & cx2_1: $8.136 \times 10^{-3}, \mathrm{cx} 2 \_3: 7.404 \times 10^{-3}$ \\
\hline Q3 & 104.360 & 23.284 & 4.856 & $1.667 \times 10^{-2}$ & $2.633 \times 10^{-4}$ & cx3_2: $7.404 \times 10^{-3}, \mathrm{cx} 3 \_4: 1.331 \times 10^{-2}$ \\
\hline Q4 & 85.217 & 87.416 & 5.117 & $1.000 \times 10^{-2}$ & $2.970 \times 10^{-4}$ & cx4_3: $1.331 \times 10^{-2}$ \\
\hline
\end{tabular}
$|j\rangle$ (i.e., the states that are actually obtained due to noise), or concisely $\mathcal{P}(i \mid j)$ where $i, j$ are $N_{q}$-qubit bit string. For

TABLE II. Noise model parameters for $i b m q \_a t h e n s$. Data for calibration date of 07/13/2020 06:13:48 GMT+02.00 (CET). In Fig. 4 the noise model corresponding to these data is denoted by "full noise." "Half noise" designates the same noise model but with halved error rates. The notation cx0_1 denotes a CNOT gate between qubits 0 (control) and 1 (target). 

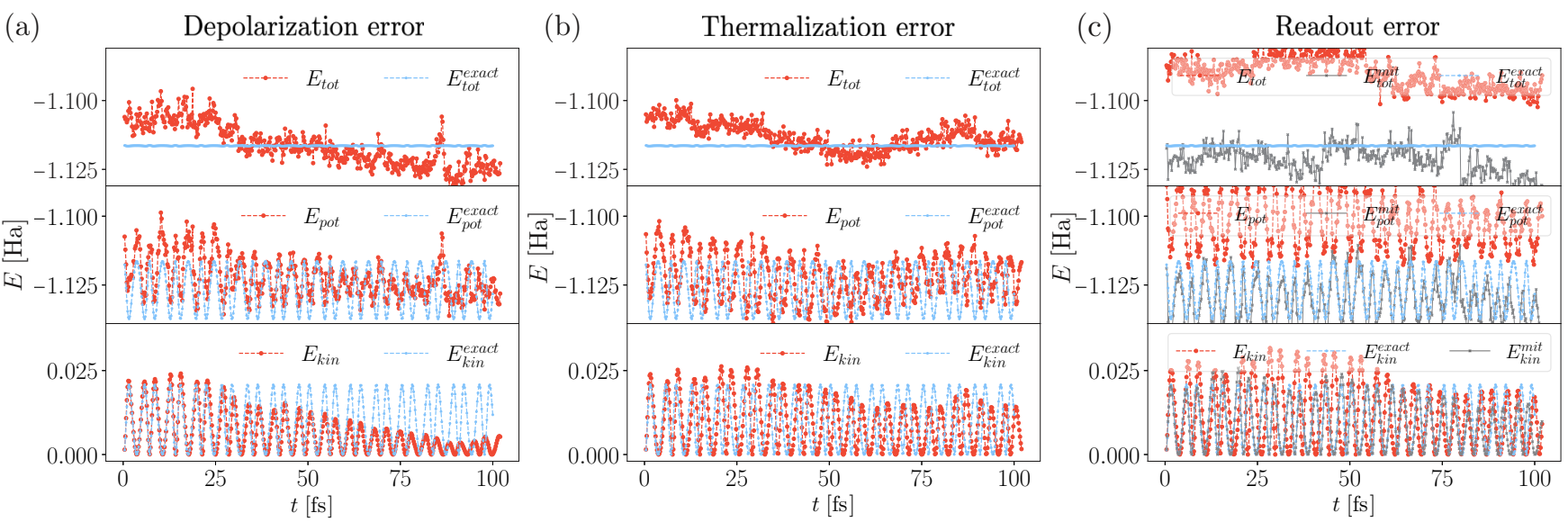

FIG. 9. Evolution of the total, potential, and kinetic energies for the dynamics of the $\mathrm{H}_{2}$ molecule $(t=100 \mathrm{fs}, d t=0.2 \mathrm{fs})$. Using the VQE algorithm and the noise model of $i b m q$ athens chip with 8192 shots we demonstrate the effects of individual noise components, namely (a) depolarization, (b) thermalization, and (c) readout errors. In all panels the blue curves correspond to the reference exact dynamics obtained with the MR. In gray, we show the results of the simulations with mitigated readout errors. Details of the calculations are described in Sec. IV and Appendix C.

instance, in an ideal noiseless situation, this matrix would be characterized by its matrix elements $\mathcal{P}(i \mid j)=1$ for $i=j$ and $\mathcal{P}(i \mid j)=0$ for $i \neq j$.

To identify the major causes for the decay of kinetic energy in the $\mathrm{H}_{2}$ simulations with noise [see Fig. 4(a)], we investigate the individual contributions of the different noise sources. To this end, we perform a new series of MD simulations in which we activate a single noise source at a time (modeled from the ibmq_athens chip) reporting the results in Fig. 9. The settings remain the same as for the microcanonical simulations of $\mathrm{H}_{2}$ reported in Sec. IV. The reference values obtained with the MR approach are in blue. In Fig. 9(a) we observe that the depolarization error contributes the most to the loss of kinetic energy. The same is true for the total energy for which no recovery is observed over the entire simulation length (100 fs). The total energy loss amounts to about $10 \mathrm{mHa}$ every $50 \mathrm{fs}$. The thermalization error (Fig. 9(b)) has a significantly smaller impact than the depolarization error on the decay of kinetic energy, while the potential energies follows quite closely the reference profile. For the case of the readout error, the potential energy presents a significant shift in order of $20 \mathrm{mHa}$ (Fig. 9(c)). The use of measurement error mitigation corrects this issue (Fig. 9(c), gray curve) and provides PES and forces that are significantly improved, in better agreement with the exact reference.

\section{APPENDIX D: SELECTION OF THE LANCZOS PARAMETER}

In this section, we provide information about the selection of the parameter $d$ in the Lanczos approach. To this end, we perform a scan in the parameter $d$ for the system energy using 8192 measurement (shots). For the case of the $\mathrm{H}_{2}$ molecule, the test is repeated for three different bond distances: 0.6, 0.7, and $0.9 \AA$ (see Fig. 10). All other simulation parameters are specified in Sec. IV. We observe that from $d>-0.75$ the error on the energy $\Delta E$ (namely the difference between the actual measurement on the hardware and the exact energy value obtained from the diagonalization of Hamiltonian) start to increase linearly. Smaller values of $d$ (i.e., $d<-0.75$ ) leads to significant increase in the error on the energy at all bond distances together with an increase of the standard deviation associated with it, $\sigma(E)$. In order to guarantee accurate simulations for the ensemble or structures sampled during $\mathrm{MD}$, we decided to take an intermediate value for $d$. Based on Fig. 10, we selected a value of $d_{\mathrm{PES}}=-0.4$, which produces accurate energy values at all geometries. Note that this value is specific for the calculation of the energy expectation values
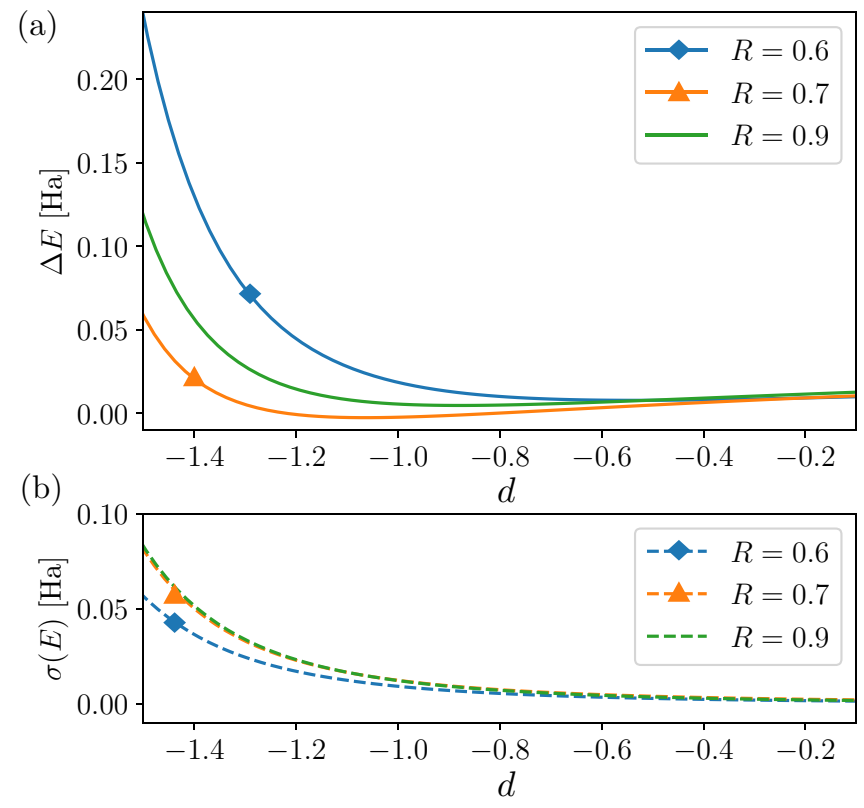

FIG. 10. (a) Error $\Delta E$ and (b) standard deviation $\sigma(E)$ of the energy expectation value computed with 8192 shots for $\mathrm{H}_{2}$ molecule with bond lengths $R=0.6,0.7$, and $0.9 \AA$ using the Lanczos method with different values of parameter $d$. 
for potential energy surfaces (from which the subscript PES in $\left.d_{\mathrm{PES}}\right)$. A similar approach is needed to select the corresponding optimal $d$ value for the forces calculations. The same procedure is then also applied to the case of the $\mathrm{H}_{3}^{+}$molecule. It is important to mention that the use of the error mitigation scheme does not obviously require the a priori knowledge of the exact energy to set the value of the parameter $d$. In general, the parameter $d$ can be set equal to the Hartree-Fock energy (i.e., $d=E_{\mathrm{HF}}$ ). From this initial guess, $d=E_{\mathrm{HF}}$, one can further optimize the correction by gradually increasing the value of $d$ until a sufficiently small standard deviation $\sigma(E)$ is reached (see Fig. 10). Note that in the limit of $d \rightarrow+\infty$ the energy estimate converges to the original noisy value.

\section{APPENDIX E: REDUCTION OF QUANTUM RESOURCES}

For the case of $\mathrm{H}_{2}$, to reduce the required quantum resources, the fermionic operators $\hat{a}_{r}^{\dagger}, \hat{a}_{i}$ in the molecular Hamiltonian $\hat{H}(\boldsymbol{R})$ are mapped to Pauli strings $P_{\lambda}$ [see Eq. (3)] using the parity transformation [70]. This fermion-to-qubit mapping allows us to exploit the particle-number symmetry $\left[\hat{H}(\boldsymbol{R}), \hat{N}_{\uparrow}\right]=\left[\hat{H}(\boldsymbol{R}), \hat{N}_{\downarrow}\right]=0$ with $\hat{N}_{\sigma}$ being the number operator for electrons of spin $\sigma \in\{\uparrow, \downarrow\}$. This technique is a part of the "tapering off" procedure [70] which permits the elimination of a qubit for each symmetry found in the Pauli string representation of the Hamiltonian. Hence, as the commutators described right above correspond to two symmetries, two qubits can be eliminated from the simulation without modifying the spectrum of the Hamiltonian. For the detailed explanation, we refer to the original work of Bravyi et al. [70] and, in context of quantum chemistry, see Appendix F in Ref. [18].

In the $\mathrm{H}_{3}^{+}$simulations, we opt for the seniority-zero Hamiltonian approximation (or doubly occupied configuration interaction Hamiltonian) [71-73] that further reduces the required computational resources. For its implementation we follow closely the steps and report the equations provided in the work of Elfving et al. [73].

In essence, only half of the orbitals are required for the computation of the ground state of the systems considered in this paper, bringing the advantage of halving the total number of qubits. However, in this approach, the full Hilbert space is restricted only to the subspace of electronic states in which the orbitals are either empty or occupied by a pair of electrons (i.e., singlet states) limiting the applicability of this method.

Note that for the two-electron systems the seniority-zero Hamiltonian becomes exact when referring to the optimal orbital basis (i.e., exact natural orbitals) which differs from the RHF orbital basis used in this work. For instance, in the case of closed-shell molecules, the corresponding seniority-zero wave function is of Löwdin-Shull type [80]. In general, the use of RHF orbitals, as in this work, makes the seniorityzero formalism approximate. For example, in the regime of strong correlation, the seniority-zero approximation misses the dynamical correlations and the results can only provide a qualitative description (see results for $\mathrm{N}_{2}, \mathrm{CO}_{2}$, and $\mathrm{H}_{8}$ molecules in Ref. [71]). However, the systems studied in this work $\left(\mathrm{H}_{2}\right.$ and $\left.\mathrm{H}_{3}^{+}\right)$do not exhibit strong correlation effects and therefore the use of HF spin orbitals is appropriate.
The starting point is the seniority-zero Hamiltonian that can be written as

$$
\hat{H}_{\mathrm{S}-0}(\boldsymbol{R})=\sum_{r s} h_{r s}^{*}(\boldsymbol{R}) \hat{b}_{r}^{\dagger} \hat{b}_{s}+\sum_{r \neq s} g_{r s}^{*}(\boldsymbol{R}) \hat{b}_{r}^{\dagger} \hat{b}_{r} \hat{b}_{s}^{\dagger} \hat{b}_{s}+E_{N N}(\boldsymbol{R}),
$$

where, differently from its unrestricted version (Eq. (1)), the $\hat{b}_{r}^{\dagger}, \hat{b}_{r}$ are the creation and annihilation operators of a pair of electrons in the mode $r$. More specifically, the indices $r$ and $s$ label the general (occupied or unoccupied) molecular orbitals.

This operator respects the following hard-core boson (anti-)commutation relations:

$$
\begin{aligned}
& {\left[\hat{b}_{r}, \hat{b}_{s}^{\dagger}\right]=\left[\hat{b}_{r}^{\dagger}, \hat{b}_{s}^{\dagger}\right]=\left[\hat{b}_{r}, \hat{b}_{s}\right]=0 \quad(r \neq s),} \\
& \left\{\hat{b}_{r}^{\dagger}, \hat{b}_{r}^{\dagger}\right\}=\left\{\hat{b}_{r}, \hat{b}_{r}\right\}=0, \\
& \left\{\hat{b}_{r}, \hat{b}_{r}^{\dagger}\right\}=1 .
\end{aligned}
$$

The integrals $h_{r s}^{*}(\boldsymbol{R})$ and $g_{r s}^{*}(\boldsymbol{R})$ related to the original single-/two-electron integrals (see Eqs. (A1) and (A2)) are as follows:

$$
\begin{aligned}
& h_{r r}^{*}(\boldsymbol{R})=2 h_{r r}(\boldsymbol{R})+g_{r r r r}(\boldsymbol{R}), \\
& h_{r s}^{*}(\boldsymbol{R})=g_{r r s s}(\boldsymbol{R}) \quad(r \neq s), \\
& g_{r s}^{*}(\boldsymbol{R})=2 g_{r s s r}(\boldsymbol{R})-g_{r s r s}(\boldsymbol{R}) \quad(r \neq s) .
\end{aligned}
$$

For additional details see also Ref. [72]. Using the fact that the operators $\hat{b}_{r}^{\dagger}, \hat{b}_{r}$ commute, they can be written using Pauli

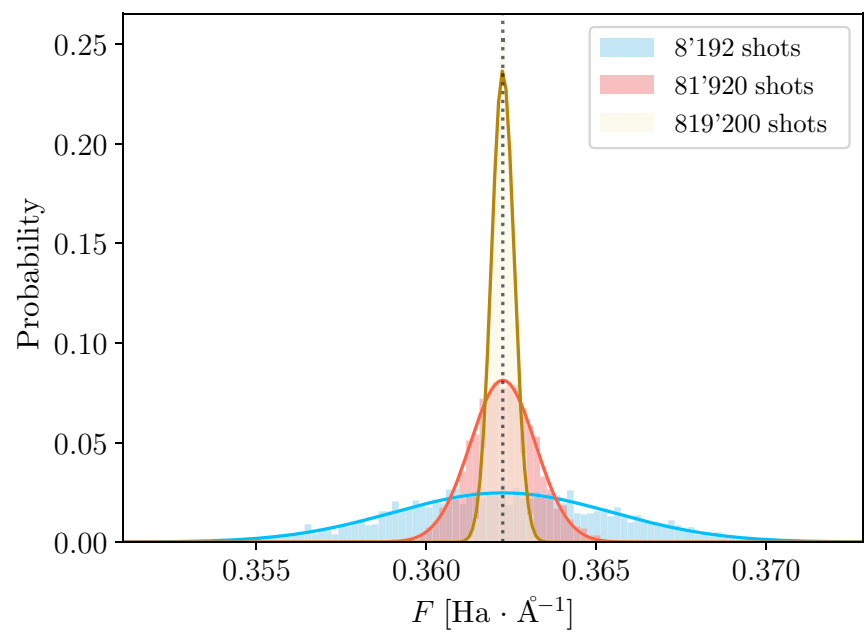

FIG. 11. Normalized distributions of the expectation values of the nuclear forces in $\mathrm{H}_{2}$ at the bond length $R=0.6 \AA$ evaluated for different number of measurements: 8192,81920 and 819200 with the VQE approach. All calculations were performed using the same setup outlined in Sec. IV of the main text. The distributions are fitted with the Gaussian function $\rho(F)=a e^{-\left(F-F_{e}\right)^{2} / 2 \sigma^{2}}$ where $F_{e}$ is the exact force obtained with FD using Eq. (6) and $\Delta R=0.001 \AA$; this value also corresponds to the center of the distributions marked with the vertical dashed line. The fitting parameters for the three different statistics are $a: 2.4 \times 10^{-2}, 8.1 \times 10^{-2}, 2.3 \times 10^{-1}, F_{e}: 0.362$, $0.362,0.362\left(\mathrm{Ha}^{-1}\right)$, and $\sigma: 3.2 \times 10^{-3}, 9.0 \times 10^{-4}, 3.0 \times 10^{-4}$ $\left(\mathrm{Ha} \AA^{-1}\right)$, respectively. 
operators [see Eq. (B1)]

$$
\hat{b}_{r}=\frac{1}{2}\left(\hat{\sigma}_{r}^{x}-i \hat{\sigma}_{r}^{y}\right) .
$$

Note that a similar mapping can also be found in the context of model pairing Hamiltonians (e.g., for superconductors) leading also, in this case, to a reduction of the number of qubits needed for the mapping [74]. After the substitution of Eq. (E3) into Eq. (E1), the qubit Hamiltonian in Pauli representation reads as

$$
\begin{aligned}
\hat{H}_{\mathrm{S}-0}^{q}= & \sum_{r} \frac{h_{r r}^{*}(\boldsymbol{R})}{2}\left(\hat{I}_{r}-\hat{\sigma}_{r}^{z}\right)+\sum_{r \neq s} \frac{h_{r s}^{*}(\boldsymbol{R})}{4}\left(\hat{\sigma}_{r}^{x} \hat{\sigma}_{s}^{x}+\hat{\sigma}_{r}^{y} \hat{\sigma}_{s}^{y}\right) \\
& +\sum_{r \neq s} \frac{g_{r s}^{*}(\boldsymbol{R})}{4}\left(\hat{I}_{r}-\hat{\sigma}_{r}^{z}-\hat{\sigma}_{s}^{z}+\hat{\sigma}_{r}^{z} \hat{\sigma}_{s}^{z}\right) \\
& +E_{N N}(\boldsymbol{R}) .
\end{aligned}
$$

To summarize, for the simulations of the $\mathrm{H}_{2}$ molecule we employ the parity transformation with two-qubit reduction, hence, the simulations require in total two qubits. Note that we do not consider for $\mathrm{H}_{2}$ further reductions to a single qubit problem as we are mainly interested on noise effects associated to two-qubit gates (CNOTs). For $\mathrm{H}_{3}^{+}$we first employ the seniority-zero formalism to reduce the problem size to three qubits. In a second step, one further qubit is tapered off leading to a final two qubit Hamiltonian. The use of the seniority-zero formalism is justified by the fact that the dynamics only samples bonded molecular configurations.

\section{APPENDIX F: THE RY ANSATZ}

For the implementation of the RY ansatz, the gate $U 3(\theta, \phi, \lambda)$ is defined as

$$
U 3(\theta, \phi, \lambda)=\left(\begin{array}{cc}
\cos \left(\frac{\theta}{2}\right) & -e^{i \lambda} \sin \left(\frac{\theta}{2}\right) \\
e^{i \phi} \sin \left(\frac{\theta}{2}\right) & e^{i(\phi+\lambda)} \cos \left(\frac{\theta}{2}\right)
\end{array}\right),
$$

and the gate $U 2(\phi, \lambda)$ is written as

$$
U 2(\phi, \lambda)=\frac{1}{\sqrt{2}}\left(\begin{array}{cc}
1 & -e^{i \lambda} \\
e^{i \phi} & e^{i(\phi+\lambda)}
\end{array}\right),
$$

where $\theta, \phi, \lambda$ are the Euler angles.

\section{APPENDIX G: MEASUREMENT NOISE STATISTICS}

In this Appendix, we show by means of simulations that the measurement noise on the nuclear forces is Gaussian distributed. To this end, we computed the magnitude of the Hellmann-Feynman forces (see Eq. (7)) along the molecular axis of $\mathrm{H}_{2}$ at the fixed bond length $R=0.6 \AA$ (without loss of generality). All other parameters are set as described in Sec. IV. We constructed three data sets corresponding to different number of measurements for the evaluation of the expectation value of the forces, namely 8192,81920 and 819200 shots. For each data set, we repeated the result of $2000 \mathrm{VQE}$ simulations each performed starting from a different random seed. These calculations led to the Gaussian-like distributions reported in Fig. 11. We also observe the typical narrowing of the distributions (peaked at the average (exact) value $F_{e}=0.362 \mathrm{Ha}^{-1}$ ) with the increase of the number of measurements.
[1] N. Moll, P. Barkoutsos, L. S. Bishop, J. M. Chow, A. Cross, D. J. Egger, S. Filipp, A. Fuhrer, J. M. Gambetta, M. Ganzhorn et al., Quantum optimization using variational algorithms on near-term quantum devices, Quantum Sci. Technol. 3, 030503 (2018).

[2] Y. Cao, J. Romero, J. P. Olson, M. Degroote, P. D. Johnson, M. Kieferová, I. D. Kivlichan, T. Menke, B. Peropadre, N. P. Sawaya et al., Quantum chemistry in the age of quantum computing, Chem. Rev. 119, 10856 (2019).

[3] S. McArdle, S. Endo, A. Aspuru-Guzik, S. C. Benjamin, and X. Yuan, Quantum computational chemistry, Rev. Mod. Phys. 92, 015003 (2020).

[4] S. McArdle, A. Mayorov, X. Shan, S. Benjamin, and X. Yuan, Digital quantum simulation of molecular vibrations, Chem. Sci. 10, 5725 (2019).

[5] N. P. Sawaya, T. Menke, T. H. Kyaw, S. Johri, A. Aspuru-Guzik, and G. G. Guerreschi, Resource-efficient digital quantum simulation of d-level systems for photonic, vibrational, and spin-s Hamiltonians, npj Quantum Inf. 6, 1 (2020).

[6] P. J. Ollitrault, A. Baiardi, M. Reiher, and I. Tavernelli, Hardware efficient quantum algorithms for vibrational structure calculations, Chem. Sci. 11, 6842 (2020).

[7] A. Peruzzo, J. McClean, P. Shadbolt, M.-H. Yung, X.-Q. Zhou, P. J. Love, A. Aspuru-Guzik, and J. L. O'brien, A variational eigenvalue solver on a photonic quantum processor, Nat. Commun. 5, 4213 (2014).
[8] A. Kandala, A. Mezzacapo, K. Temme, M. Takita, M. Brink, J. M. Chow, and J. M. Gambetta, Hardware-efficient variational quantum eigensolver for small molecules and quantum magnets, Nature (London) 549, 242 (2017).

[9] M. Ganzhorn, D. J. Egger, P. Barkoutsos, P. Ollitrault, G. Salis, N. Moll, M. Roth, A. Fuhrer, P. Mueller, S. Woerner et al., GateEfficient Simulation of Molecular Eigenstates on a Quantum Computer, Phys. Rev. Appl. 11, 044092 (2019).

[10] P. J. J. OMalley, R. Babbush, I. D. Kivlichan, J. Romero, J. R. McClean, R. Barends, J. Kelly, P. Roushan, A. Tranter, N. Ding, B. Campbell, Y. Chen, Z. Chen, B. Chiaro, A. Dunsworth, A. G. Fowler, E. Jeffrey, E. Lucero, A. Megrant, J. Y. Mutus, M. Neeley, C. Neill, C. Quintana, D. Sank, A. Vainsencher, J. Wenner, T. C. White, P. V. Coveney, P. J. Love, H. Neven, A. Aspuru-Guzik, and J. M. Martinis, Scalable Quantum Simulation of Molecular Energies, Phys. Rev. X 6, 031007 (2016).

[11] P. K. Barkoutsos, J. F. Gonthier, I. Sokolov, N. Moll, G. Salis, A. Fuhrer, M. Ganzhorn, D. J. Egger, M. Troyer, A. Mezzacapo et al., Quantum algorithms for electronic structure calculations: Particle-hole Hamiltonian and optimized wave-function expansions, Phys. Rev. A 98, 022322 (2018).

[12] C. Hempel, C. Maier, J. Romero, J. McClean, T. Monz, H. Shen, P. Jurcevic, B. P. Lanyon, P. Love, R. Babbush et al., Quantum Chemistry Calculations on a Trapped-Ion Quantum Simulator, Phys. Rev. X 8, 031022 (2018). 
[13] R. Santagati, J. Wang, A. A. Gentile, S. Paesani, N. Wiebe, J. R. McClean, S. Morley-Short, P. J. Shadbolt, D. Bonneau, J. W. Silverstone et al., Witnessing eigenstates for quantum simulation of hamiltonian spectra, Sci. Adv. 4, eaap9646 (2018).

[14] J. I. Colless, V. V. Ramasesh, D. Dahlen, M. S. Blok, M. Kimchi-Schwartz, J. R. McClean, J. Carter, W. A. de Jong, and I. Siddiqi, Computation of Molecular Spectra on a Quantum Processor with an Error-Resilient Algorithm, Phys. Rev. X 8, 011021 (2018).

[15] A. Kandala, K. Temme, A. D. Córcoles, A. Mezzacapo, J. M. Chow, and J. M. Gambetta, Error mitigation extends the computational reach of a noisy quantum processor, Nature (London) 567, 491 (2019).

[16] M. Kühn, S. Zanker, P. Deglmann, M. Marthaler, and H. Weiß, Accuracy and resource estimations for quantum chemistry on a near-term quantum computer, J. Chem. Theory Comput. 15, 4764 (2019).

[17] G. A. Quantum et al., Hartree-Fock on a superconducting qubit quantum computer, Science 369, 1084 (2020).

[18] I. O. Sokolov, P. K. Barkoutsos, P. J. Ollitrault, D. Greenberg, J. Rice, M. Pistoia, and I. Tavernelli, Quantum orbital-optimized unitary coupled cluster methods in the strongly correlated regime: Can quantum algorithms outperform their classical equivalents?, J. Chem. Phys. 152, 124107 (2020).

[19] H. R. Grimsley, S. E. Economou, E. Barnes, and N. J. Mayhall, An adaptive variational algorithm for exact molecular simulations on a quantum computer, Nat. Commun. 10, 1 (2019).

[20] Y. Shen, X. Zhang, S. Zhang, J.-N. Zhang, M.-H. Yung, and K. Kim, Quantum implementation of the unitary coupled cluster for simulating molecular electronic structure, Phys. Rev. A 95, 020501(R) (2017).

[21] A. Y. Kitaev, Quantum computations: Algorithms and error correction, Russ. Math. Surv. 52, 1191 (1997).

[22] A. J. C. Woitzik, P. K. Barkoutsos, F. Wudarski, A. Buchleitner, and I. Tavernelli, Entanglement production and convergence properties of the variational quantum eigensolver, Phys. Rev. A 102, 042402 (2020).

[23] A. Aspuru-Guzik, A. D. Dutoi, P. J. Love, and M. Head-Gordon, Simulated quantum computation of molecular energies, Science 309, 1704 (2005).

[24] R. Babbush, N. Wiebe, J. McClean, J. McClain, H. Neven, and G. K.-L. Chan, Low-Depth Quantum Simulation of Materials, Phys. Rev. X 8, 011044 (2018).

[25] L. M. Milne-Thomson, The Calculus of Finite Differences (American Mathematical Society, Providence, RI, 2000).

[26] I. Kassal and A. Aspuru-Guzik, Quantum algorithm for molecular properties and geometry optimization, J. Chem. Phys. 131, 224102 (2009).

[27] K. Mitarai, Y. O. Nakagawa, and W. Mizukami, Theory of analytical energy derivatives for the variational quantum eigensolver, Phys. Rev. Res. 2, 013129 (2020).

[28] T. E. O'Brien, B. Senjean, R. Sagastizabal, X. Bonet-Monroig, A. Dutkiewicz, F. Buda, L. DiCarlo, and L. Visscher, Calculating energy derivatives for quantum chemistry on a quantum computer, npj Quantum Inf. 5, 113 (2019).

[29] R. M. Parrish, E. G. Hohenstein, P. L. McMahon, and T. J. Martinez, Hybrid quantum/classical derivative theory: Analytical gradients and excited-state dynamics for the multistate contracted variational quantum eigensolver, arXiv:1906.08728.
[30] M. P. Allen and D. J. Tildesley, Computer Simulation of Liquids (Clarendon, London, 1987).

[31] C. Attaccalite and S. Sorella, Stable Liquid Hydrogen at High Pressure by a Novel Ab Initio Molecular-Dynamics Calculation, Phys. Rev. Lett. 100, 114501 (2008).

[32] T. D. Kühne, M. Krack, F. R. Mohamed, and M. Parrinello, Efficient and Accurate Car-Parrinello-Like Approach to BornOppenheimer Molecular Dynamics, Phys. Rev. Lett. 98, 066401 (2007).

[33] K. Temme, S. Bravyi, and J. M. Gambetta, Error Mitigation for Short-Depth Quantum Circuits, Phys. Rev. Lett. 119, 180509 (2017).

[34] Y. Li and S. C. Benjamin, Efficient Variational Quantum Simulator Incorporating Active Error Minimization, Phys. Rev. X 7, 021050 (2017).

[35] S. Endo, S. C. Benjamin, and Y. Li, Practical Quantum Error Mitigation for Near-Future Applications, Phys. Rev. X 8 031027 (2018).

[36] E. F. Dumitrescu, A. J. McCaskey, G. Hagen, G. R. Jansen, T. D. Morris, T. Papenbrock, R. C. Pooser, D. J. Dean, and P. Lougovski, Cloud Quantum Computing of an Atomic Nucleus, Phys. Rev. Lett. 120, 210501 (2018).

[37] C. Lanczos, An Iteration Method for the Solution of the Eigenvalue Problem of Linear Differential and Integral Operators (U.S. GPO, Los Angeles, CA, 1950), Vol. 45.

[38] R. Mises and H. Pollaczek-Geiringer, Praktische Verfahren der Gleichungsauflösung, ZAMM-J. Appl. Math. Mechan./Z. Angew. Math. Mech. 9, 58 (1929).

[39] M. Born and R. Oppenheimer, Zur quantentheorie der molekeln, Ann. Phys. 389, 457 (1927).

[40] J. R. McClean, J. Romero, R. Babbush, and A. Aspuru-Guzik, The theory of variational hybrid quantum-classical algorithms, New J. Phys. 18, 023023 (2016).

[41] G. Torlai, G. Mazzola, G. Carleo, and A. Mezzacapo, Precise measurement of quantum observables with neural-network estimators, Phys. Rev. Res. 2, 022060 (2020).

[42] J. M. Kübler, A. Arrasmith, L. Cincio, and P. J. Coles, An adaptive optimizer for measurement-frugal variational algorithms, Quantum 4, 263 (2020).

[43] T.-C. Yen, V. Verteletskyi, and A. F. Izmaylov, Measuring all compatible operators in one series of single-qubit measurements using unitary transformations, J. Chem. Theory Comput. 16, 2400 (2020).

[44] A. F. Izmaylov, T.-C. Yen, R. A. Lang, and V. Verteletskyi, Unitary partitioning approach to the measurement problem in the variational quantum eigensolver method, J. Chem. Theory Comput. 16, 190 (2019).

[45] O. Crawford, B. van Straaten, D. Wang, T. Parks, E. Campbell, and S. Brierley, Efficient quantum measurement of pauli operators, Quantum 5, 385 (2021).

[46] P. Jordan and E. Wigner, Über das paulische äquivalenzverbot, Z. Phys. 47, 631 (1928).

[47] P. Pulay, Ab initio calculation of force constants and equilibrium geometries in polyatomic molecules: I. Theory, Mol. Phys. 17, 197 (1969).

[48] K. Mitarai and K. Fujii, Methodology for replacing indirect measurements with direct measurements, Phys. Rev. Res. 1, 013006 (2019). 
[49] P. Güttinger, Das verhalten von atomen im magnetischen drehfeld, Z. Phys. 73, 169 (1932).

[50] W. Pauli, Die allgemeinen Prinzipien der Wellenmechanik, Quantentheorie (Springer Berlin Heidelberg, Berlin, 1933), pp. 83-272.

[51] H. Hellman, Einführung in Die Quantenchemie (Franz Deuticke, Leipzig, 1937), p. 285.

[52] R. P. Feynman, Forces in molecules, Phys. Rev. 56, 340 (1939).

[53] C. Filippi and C. Umrigar, Correlated sampling in quantum Monte Carlo: A route to forces, Phys. Rev. B 61, R16291 (2000).

[54] T. U. Helgaker and J. Almlöf, A second-quantization approach to the analytical evaluation of response properties for perturbation-dependent basis sets, Int. J. Quantum Chem. 26, 275 (1984).

[55] M. A. Nielsen, I. L. Chuang, Quantum Computation and Quantum Information: 10th Anniversary Edition, 10th ed. (Cambridge University Press, New York, 2011).

[56] G. Aleksandrowicz, T. Alexander, P. Barkoutsos, L. Bello, Y. Ben-Haim, D. Bucher, F. J. Cabrera-Hernandez, J. CarballoFranquis, A. Chen, C.-F. Chen, J. M. Chow, A. D. CorcolesGonzales, A. J. Cross, A. Cross, J. Cruz-Benito, C. Culver, S. D. L. P. Gonzalez, E. D. L. Torre, D. Ding, E. Dumitrescu, I. Duran, P. Eendebak, M. Everitt, I. F. Sertage, A. Frisch, A. Fuhrer, J. Gambetta, B. G. Gago, J. Gomez-Mosquera, D. Greenberg, I. Hamamura, V. Havlicek, J. Hellmers, L. Herok, H. Horii, S. Hu, T. Imamichi, T. Itoko, A. Javadi-Abhari, N. Kanazawa, A. Karazeev, K. Krsulich, P. Liu, Y. Luh, Y. Maeng, M. Marques, F. J. Martin-Fernandez, D. T. McClure, D. McKay, S. Meesala, A. Mezzacapo, N. Moll, D. M. Rodriguez, G. Nannicini, P. Nation, P. J. Ollitrault, L. J. O'Riordan, H. Paik, J. Perez, A. Phan, M. Pistoia, V. Prutyanov, M. Reuter, J. Rice, A. R. Davila, R. H. P. Rudy, M. Ryu, N. Sathaye, C. Schnabel, E. Schoute, K. Setia, Y. Shi, A. Silva, Y. Siraichi, S. Sivarajah, J. A. Smolin, M. Soeken, I. Sokolov, H. Takahashi, I. Tavernelli, C. Taylor, P. Taylour, K. Trabing, M. Treinish, W. Turner, D. Vogt-Lee, C. Vuillot, J. A. Wildstrom, J. Wilson, E. Winston, C. Wood, S. Wood, S. Wörner, I. Y. Akhalwaya, C. Zoufal, Qiskit: An open-source framework for quantum computing, 2019, doi: 10.5281/zenodo.2562111.

[57] P. Suchsland, F. Tacchino, M. H. Fischer, T. Neupert, P. K. Barkoutsos, and I. Tavernelli, Algorithmic error mitigation scheme for current quantum processors, arXiv:2008.10914.

[58] K. Seki and S. Yunoki, Quantum power method by a superposition of time-evolved states, arXiv:2008.03661.

[59] The notation $\boldsymbol{X} / \boldsymbol{m}$, where $\boldsymbol{X}$ is a $3 N$-dimensional vector like the velocity $\boldsymbol{v}$ or the force $\boldsymbol{F}$ vector, implies that the component $X_{I \alpha}$ of the vector $\boldsymbol{X}$ corresponding to the $I$ th ion and $\alpha$ th direction, is divided by the mass $m_{I}$ of the $I$ th ion.

[60] L. Verlet, Computer "experiments" on classical fluids. I. Thermodynamical properties of Lennard-Jones molecules, Phys. Rev. 159, 98 (1967).

[61] G. Bussi and M. Parrinello, Accurate sampling using Langevin dynamics, Phys. Rev. E 75, 056707 (2007).

[62] G. Mazzola, S. Yunoki, and S. Sorella, Unexpectedly high pressure for molecular dissociation in liquid hydrogen by electronic simulation, Nat. Commun. 5, 3487 (2014).
[63] Y. Luo, A. Zen, and S. Sorella, Ab initio molecular dynamics with noisy forces: Validating the quantum Monte Carlo approach with benchmark calculations of molecular vibrational properties, J. Chem. Phys. 141, 194112 (2014).

[64] G. Mazzola, A. Zen, and S. Sorella, Finite-temperature electronic simulations without the Bborn-Oppenheimer constraint, J. Chem. Phys. 137, 134112 (2012).

[65] G. Mazzola and S. Sorella, Accelerating Ab Initio Molecular Dynamics and Probing the Weak Dispersive Forces in Dense Liquid Hydrogen, Phys. Rev. Lett. 118, 015703 (2017).

[66] G. Mazzola, R. Helled, and S. Sorella, Phase Diagram of Hydrogen and a Hydrogen-Helium Mixture at Planetary Conditions by Quantum Monte Carlo Simulations, Phys. Rev. Lett. 120, 025701 (2018).

[67] M. Rossi, V. Kapil, and M. Ceriotti, Fine tuning classical and quantum molecular dynamics using a generalized Langevin equation, J. Chem. Phys. 148, 102301 (2018).

[68] F. Becca, S. Sorella, Quantum Monte Carlo Approaches for Correlated Systems (Cambridge University Press, Cambridge, 2017).

[69] M. Ceriotti, G. Bussi, and M. Parrinello, Langevin Equation with Colored Noise for Constant-Temperature Molecular Dynamics Simulations, Phys. Rev. Lett. 102, 020601 (2009).

[70] S. Bravyi, J. M. Gambetta, A. Mezzacapo, and K. Temme, Tapering off qubits to simulate fermionic Hamiltonians, arXiv:1701.08213.

[71] L. Bytautas, T. M. Henderson, C. A. Jiménez-Hoyos, J. K. Ellis, and G. E. Scuseria, Seniority and orbital symmetry as tools for establishing a full configuration interaction hierarchy, J. Chem. Phys. 135, 044119 (2011).

[72] P. A. Limacher, A new wavefunction hierarchy for interacting geminals, J. Chem. Phys. 145, 194102 (2016).

[73] V. E. Elfving, J. A. Gámez, and C. Gogolin, Simulating quantum chemistry in the restricted Hartree-Fock space on a qubit-based quantum computing device, arXiv:2002.00035.

[74] A. Khamoshi, F. Evangelista, and G. E. Scuseria, Correlating AGP on a quantum computer, Quantum Sci. Technol. 6, 014004 (2020).

[75] W. Lavrijsen, A. Tudor, J. Müller, C. Iancu, and W. de Jong, Classical optimizers for noisy intermediate-scale quantum devices, in 2020 IEEE International Conference on Quantum Computing and Engineering (QCE) (Denver, CO, USA, 2020), pp. 267-277.

[76] P. Virtanen, R. Gommers, T. E. Oliphant, M. Haberland, T. Reddy, D. Cournapeau, E. Burovski, P. Peterson, W. Weckesser, J. Bright et al., Scipy 1.0: Fundamental algorithms for scientific computing in Python, Nat. Methods 17, 261 (2020).

[77] M. Rossmannek, P. K. Barkoutsos, P. J. Ollitrault, and I. Tavernelli, Quantum hf/dft-embedding algorithms for electronic structure calculations: Scaling up to complex molecular systems, arXiv:2009.01872.

[78] https://www.ibm.com/legal/copytrade.

[79] Q. Sun, T. C. Berkelbach, N. S. Blunt, G. H. Booth, S. Guo, Z. Li, J. Liu, J. D. McClain, E. R. Sayfutyarova, S. Sharma et al., PySCF: the Python-based simulations of chemistry framework, WIREs Comput. Mol. Sci. 8, e1340 (2018).

[80] P.-O. Löwdin and H. Shull, Natural orbitals in the quantum theory of two-electron systems, Phys. Rev. 101, 1730 (1956). 